\title{
An Economic Analysis of Productivity and Performance of Public Sector Banks in India
}

\author{
Geetu Gupta*, Amandeep Kaur \\ Junior Research Fellow (JRF), Punjabi University, Patiala \\ *Corresponding Author: ecogeet@gmail.com
}

Copyright (C 2013 Horizon Research Publishing All rights reserved

\begin{abstract}
The paper makes an attempt to analyze the productivity performance of public sector banks in India on the basis of Employee Productivity and Branch Productivity. The variables selected for the study is based on secondary data published by Indian Banks Association (IBA) for the period 1991-2010. Mean, standard deviation and coefficient of variation have been used to analyze the productivity. Based on the findings the banks are divided into four categories i.e. excellent, good, fair and poor for the period under study. Finally, appropriate policy suggestions are made for improvement of productivity in public sector banks in India.
\end{abstract}

Keywords Branch Productivity, Employee Productivity, India, National Banks, SBI Group.

\section{Introduction}

Efficient banking systems contribute in an extensive way for higher economic growth in any country, studies in this nature are very important for policy makers, industry leaders and many others who are reliant on the banking sector.

In India, till the eighties, the banks operated in a protected environment and the reform started in 1991. The reform process was to improve productivity and efficiency of the financial system. Banking performance is the mirror reflection of an economy.

Productivity is defined as the ratio of Output to Input. In manufacturing organizations the value added or net output is taken as the output measure. In the service sector it is difficult to quantify the output because it is intangible. Hence, different proxy indicators are used for measuring productivity of service organizations. The indicators commonly used for assessing productivity of banks are Business per employee/ Branch, advances per employee/Branch, number of accounts per employee/branch etc. In our study we have used Deposits per employee, Advances per employee, Net Profit per employee, Deposits per branch, Advances per Branch and Net profit per branch as indicators of productivity of banks. The sample used for the study consists of 27 public sector banks in which nationalized banks (19) and SBI \& its associates (7).

\section{Objectives of the Study}

The main objectives of the study are as follows:-

1. To analyze the performance of public sector banks in India.

2. To study about the productivity in public sector banks in India.

3. To suggest measures for improvement of financial performance of banks.

\section{Literature Review}

Shanmugam and Das(2004) measured the technical efficiency of banks in four different ownership groups in India during the reform period 1992-1999. In this study variables of outputs taken are net interest margin, non-interest income, credits and investments. The inputs used are deposits, borrowings, labor and fixed assets. The data set is an unbalanced panel of 94 banks belonging to different ownership groups using stochastic frontier approach for panel data. The result shows the dominance of deposit in producing all outputs. The technical efficiency of raising interest margin is varied widely across sample banks and is time-invariant. The several reform measures have not so far helped the banks in raising their interest margin. However, the banking sector shows a progress in terms of efficiency of raising non-interest income, investments and credits. This improvement is considerable in the case of investments in all banks, particularly in private banks. Thus, the result matches with the economic growth objective of the reform measure. In case of sample banks, the result shows the state bank group and private foreign banks perform better than their counter parts.

Mohan (2005) explores the efficiency of banks during post reform period in India. There has been a consistent decline in the share of public sector banks in total assets of commercial 
banks and a declining trend of Herfindahl's concentration index. Improvements in efficiency of the banking system were reflected in a number of indicators, such as, a gradual reduction in cost of intermediation (defined as the ratio of operating expense to total assets) across various bank groups (barring foreign banks), and decline in the non-performing loans. As a result of these changes, there has been an all-around productivity improvement in the Indian banking sector. While the cost income-ratio (i.e., the ratio of operating expenses to total income less interest expense) as well as net interest margin (i.e., the excess of interest income over interest expense, scaled by total bank assets) of Indian banks showed a declining trend .The business per employee of Indian banks increased over three-fold in real terms exhibiting an annual compound growth rate of nearly 9 percent. Such productivity improvements in the banking sector could be driven by two factors: technological improvements, which expands the range of production possibilities and peer pressure among banks compels them to raise their productivity levels.

Das (2010) estimated the performance of the Indian banking sector after the initiation of financial liberalization and also aims to measure the cost efficiency of the Indian banking sector during the post reform period 1996-2007. Using Stochastic Frontier Approach (cost frontier) and RBI data for 60 Indian commercial banks in which input include interest cost, labor cost and capital cost and output include total loans, investment in securities and other investments. On the basis of panel investigation, the paper concludes that after financial liberalization there has been no significant change in the cost efficiency of the public sector banks. The finding shows a marginal decline in the cost efficiency of the public sector banks in the post reform period. A comparison among various bank groups in the post reform period shows, the domestic private banks are becoming more efficient in comparison to the public sector and the foreign banks. The study finds that after deregulation, the concentration has declined which resulted in increasing competition. The share of private and foreign banks in banking asset, deposit and credit has gone up. The profitability of all bank groups has gone up, but the foreign banks are more profitable and the public sector banks are more cost efficient than the private and the foreign banks.

The empirical literature on Indian banks examines differences in operational efficiency and profitability across private and state-owned banks as opposed to differences across foreign and domestic banks (Sabi 1996). The general perception is that public sector involvement in the banking sector blunts incentives to effectively respond to market-based reforms (Bhattacharya and Patel, 2003; Kumbhakar and Sarkar, 2003) so that deregulation benefits private banks. In the Indian context, in particular, a research agenda focused on within public sector bank performance would be a significant contribution to the literature.

\section{Data and Methodology}

The study is mainly based on secondary data drawn from the performance highlights of public sector banks published by Indian Banks Association (IBA).This data is related to years (1991-2010). For analysis of the data, two important statistical tools viz. mean and coefficient of variation has been used to arrive at conclusions in a scientific way.

\section{Research Framework -Performance Indices}

The 27 banks names are listed serially in Appendix A. The chosen time period is 1991-2010 as the reform process began in India in 1991. The sample period 1991-2010 divided into two sub periods:

(i) $1991-2000$

(ii) $2001-2010$

To analyze the performance, six productivity indices are calculated for the study period (1991-2010). The indices computed are as follows:

$$
\text { Index }=\frac{\text { Average ratio of the concerned public sector bank }}{\text { Average ratio for the aggregate of all public sector banks }}
$$

With regard to these productivity indices, the performance of the public sector banks has been assessed at four levels, i.e. excellent, good, fair and poor. For excellent performance level, the banks included are those lying at top $25 \%$ area of normal distribution, i.e. where growth index value is greater than $(\bar{X}+0.6745 \sigma)$. Good performance category the bank whose growth lies between ( $\bar{X}$ to $\bar{X}+0.6745 \sigma$ ). Fair performance category includes banks whose growth index value lies between ( $\bar{X}$ to $\bar{X}-0.6745 \sigma)$. Poor performance category includes banks whose growth index value is lower than $(\bar{X}-0.6745 \sigma)$.

In table 5.1 the bank productivity indices for 27 public sector banks measured by employee productivity and branch productivity. The maximum employee productivity indices were revealed by Oriental bank of Commerce (1.55) in deposit per employee, Bank of Baroda (1.51) in advances per employee and Corporation bank (6.8) in net profit per employee. On the other hand least productivity indices were revealed by State bank of Bikaner and Jaipur (0.71) in deposit per employee, united bank of India (0.59) in advances per employee and Indian bank (-6.35) in net profit per employee. 
Table 5.1. Indices of Productivity Performance (1991-2000)

\begin{tabular}{|c|c|c|c|c|c|c|}
\hline Name of the bank & $\mathrm{D} / \mathrm{E}$ & $\mathrm{A} / \mathrm{E}$ & $\mathrm{NP} / \mathrm{E}$ & $\mathrm{D} / \mathrm{B}$ & $\mathrm{A} / \mathrm{B}$ & $\mathrm{NP} / \mathrm{B}$ \\
\hline Allahabad bank & $0.99 \mathrm{~F}$ & $0.93 \mathrm{~F}$ & $-0.17 \mathrm{P}$ & $0.66 \mathrm{P}$ & $0.62 \mathrm{P}$ & $-0.16 \mathrm{P}$ \\
\hline Andhra bank & $1.03 \mathrm{G}$ & $0.88 \mathrm{~F}$ & $0.62 \mathrm{~F}$ & $0.85 \mathrm{~F}$ & $0.72 \mathrm{P}$ & $0.32 \mathrm{~F}$ \\
\hline Bank of Baroda & $1.35 \mathrm{E}$ & $1.51 \mathrm{E}$ & $3.07 \mathrm{E}$ & $1.36 \mathrm{E}$ & $1.51 \mathrm{E}$ & $3.15 \mathrm{E}$ \\
\hline Bank of India & $1.16 \mathrm{E}$ & $1.34 \mathrm{E}$ & $0.39 \mathrm{~F}$ & $1.35 \mathrm{E}$ & $1.54 \mathrm{E}$ & $0.33 \mathrm{~F}$ \\
\hline Bank of Maharashtra & $0.91 \mathrm{~F}$ & $0.76 \mathrm{P}$ & $-0.54 \mathrm{P}$ & $0.71 \mathrm{P}$ & $0.59 \mathrm{P}$ & $-0.68 \mathrm{P}$ \\
\hline Canara bank & $1.09 \mathrm{G}$ & $1.06 \mathrm{G}$ & $1.99 \mathrm{G}$ & $1.42 \mathrm{E}$ & $1.37 \mathrm{E}$ & $2.66 \mathrm{E}$ \\
\hline Central Bank of India & $0.87 \mathrm{~F}$ & $0.75 \mathrm{P}$ & $-0.53 \mathrm{P}$ & $0.79 \mathrm{P}$ & $0.67 \mathrm{P}$ & $-0.59 \mathrm{P}$ \\
\hline Corporation bank & $1.49 \mathrm{E}$ & $1.44 \mathrm{E}$ & $6.82 \mathrm{E}$ & $1.44 \mathrm{E}$ & $1.37 \mathrm{E}$ & $6.59 \mathrm{E}$ \\
\hline Dena bank & $1.05 \mathrm{G}$ & $1.09 \mathrm{G}$ & $-0.28 \mathrm{P}$ & $0.73 \mathrm{P}$ & $0.75 \mathrm{P}$ & $0.04 \mathrm{~F}$ \\
\hline Indian bank & $1.02 \mathrm{G}$ & $1.09 \mathrm{G}$ & $-6.35 \mathrm{P}$ & $1.03 \mathrm{G}$ & $1.09 \mathrm{G}$ & $-6.56 \mathrm{P}$ \\
\hline Indian Overseas bank & $1.07 \mathrm{G}$ & $1.07 \mathrm{G}$ & $-1.24 \mathrm{P}$ & $1.22 \mathrm{E}$ & $1.21 \mathrm{E}$ & $-1.67 \mathrm{P}$ \\
\hline Oriental Bank of Commerce & $1.55 \mathrm{E}$ & $1.5 \mathrm{E}$ & $6.11 \mathrm{E}$ & $1.48 \mathrm{E}$ & $1.42 \mathrm{E}$ & $5.94 \mathrm{E}$ \\
\hline Punjab \& Sind bank & $1.04 \mathrm{G}$ & $0.95 \mathrm{~F}$ & $-1.29 \mathrm{P}$ & $0.95 \mathrm{~F}$ & $0.86 \mathrm{~F}$ & $-1.33 \mathrm{P}$ \\
\hline Punjab National Bank & $0.89 \mathrm{~F}$ & $0.88 \mathrm{~F}$ & $1.93 \mathrm{G}$ & $0.88 \mathrm{~F}$ & $0.85 \mathrm{~F}$ & $1.87 \mathrm{E}$ \\
\hline Syndicate bank & $0.78 \mathrm{P}$ & $0.74 \mathrm{P}$ & $-0.29 \mathrm{P}$ & $0.95 \mathrm{~F}$ & $0.88 \mathrm{~F}$ & $-0.72 \mathrm{P}$ \\
\hline UCO bank & $0.75 \mathrm{P}$ & $0.7 \mathrm{P}$ & $-2.62 \mathrm{P}$ & $0.77 \mathrm{P}$ & $0.71 \mathrm{P}$ & $-2.87 \mathrm{P}$ \\
\hline Union Bank of India & $1.18 \mathrm{E}$ & $1.11 \mathrm{G}$ & $2.13 \mathrm{G}$ & $1 \mathrm{G}$ & $0.94 \mathrm{~F}$ & $1.83 \mathrm{E}$ \\
\hline United Bank of India & $0.9 \mathrm{~F}$ & $0.59 \mathrm{P}$ & $1.87 \mathrm{G}$ & $0.83 \mathrm{P}$ & $0.54 \mathrm{P}$ & $1.79 \mathrm{E}$ \\
\hline Vijaya bank & $0.93 \mathrm{~F}$ & $0.79 \mathrm{P}$ & $-0.45 \mathrm{P}$ & $0.88 \mathrm{~F}$ & $0.75 \mathrm{P}$ & $-0.49 \mathrm{P}$ \\
\hline State Bank of India & $0.98 \mathrm{~F}$ & $1.09 \mathrm{G}$ & $2.48 \mathrm{G}$ & $1.41 \mathrm{E}$ & $1.56 \mathrm{E}$ & $3.64 \mathrm{E}$ \\
\hline Bank of Bikaner \& Jaipur & $0.71 \mathrm{P}$ & $0.76 \mathrm{P}$ & $1.98 \mathrm{G}$ & $0.76 \mathrm{P}$ & $0.81 \mathrm{~F}$ & $2.13 \mathrm{E}$ \\
\hline State bank of Hyderabad & $1.01 \mathrm{G}$ & $1.09 \mathrm{G}$ & $2.77 \mathrm{E}$ & $0.96 \mathrm{~F}$ & $1.04 \mathrm{G}$ & $2.66 \mathrm{E}$ \\
\hline State bank of Indore & $0.84 \mathrm{P}$ & $0.96 \mathrm{~F}$ & $1.82 \mathrm{G}$ & $0.83 \mathrm{P}$ & $0.95 \mathrm{~F}$ & $1.8 \mathrm{E}$ \\
\hline State bank of Mysore & $0.72 \mathrm{P}$ & $0.81 \mathrm{P}$ & $1.22 \mathrm{G}$ & $0.77 \mathrm{P}$ & $0.86 \mathrm{~F}$ & $1.35 \mathrm{G}$ \\
\hline State bank of Patiala & $0.95 \mathrm{~F}$ & $1.07 \mathrm{G}$ & $3.33 \mathrm{E}$ & $1.01 \mathrm{G}$ & $1.14 \mathrm{G}$ & $3.54 \mathrm{E}$ \\
\hline State bank of Saurashtra & $0.82 \mathrm{P}$ & $0.97 \mathrm{~F}$ & $0.53 \mathrm{~F}$ & $0.95 \mathrm{~F}$ & $1.11 \mathrm{G}$ & $0.58 \mathrm{~F}$ \\
\hline State bank of Tranvancore & $0.92 \mathrm{~F}$ & $1.04 \mathrm{G}$ & $1.69 \mathrm{G}$ & $1.01 \mathrm{G}$ & $1.13 \mathrm{G}$ & $1.85 \mathrm{E}$ \\
\hline Mean & 1 & 1 & 1 & 1 & 1 & 1 \\
\hline $\mathrm{SD}$ & 0.21 & 0.24 & 2.56 & 0.25 & 0.31 & 2.65 \\
\hline
\end{tabular}

Source: Calculated

$\mathrm{D} / \mathrm{E}=$ Deposits per Employee

$\mathrm{A} / \mathrm{E}=$ Advances per Employee

$\mathrm{NP} / \mathrm{E}=$ Net Profit per Employee

$\mathrm{D} / \mathrm{B}=$ Deposits per Branch

$\mathrm{A} / \mathrm{B}=$ Advances per Branch

$\mathrm{NP} / \mathrm{B}=$ Net Profit per Branch

' $\mathrm{E}$ '= Excellent

' $\mathrm{G}$ ' $=$ Good

'F'= Fair

'P'= Poor

In branch productivity analysis, maximum productivity indices were revealed by Oriental bank of commerce (1.48) in deposits per branch, State bank of India (1.56) in advances per branch and Corporation bank (6.59) in net profit per branch. On the other hand, least performance were revealed by Allahabad bank(0.66) in deposits per branch, united bank of India (0.54) in advances per branch and Indian bank (-6.56) in net profit per branch. 
Table 5.2. Indices of Productivity Performance (2001-2010)

\begin{tabular}{|c|c|c|c|c|c|c|}
\hline Name of the bank & $\mathrm{D} / \mathrm{E}$ & $\mathrm{A} / \mathrm{E}$ & $\mathrm{NP} / \mathrm{E}$ & $\mathrm{D} / \mathrm{B}$ & $\mathrm{A} / \mathrm{B}$ & $\mathrm{NP} / \mathrm{B}$ \\
\hline Allahabad bank & $1.02 \mathrm{G}$ & $0.99 \mathrm{~F}$ & $1.1 \mathrm{G}$ & $0.76 \mathrm{P}$ & $0.75 \mathrm{P}$ & $0.81 \mathrm{~F}$ \\
\hline Andhra bank & $1.07 \mathrm{G}$ & $1.11 \mathrm{G}$ & $1.41 \mathrm{E}$ & $0.86 \mathrm{~F}$ & $0.89 \mathrm{~F}$ & $1.13 \mathrm{G}$ \\
\hline Bank of Baroda & $1.21 \mathrm{E}$ & $1.22 \mathrm{E}$ & $1.29 \mathrm{E}$ & $1.24 \mathrm{E}$ & $1.27 \mathrm{E}$ & $1.3 \mathrm{E}$ \\
\hline Bank of India & $1.11 \mathrm{G}$ & $1.19 \mathrm{E}$ & $1.06 \mathrm{G}$ & $1.21 \mathrm{E}$ & $1.31 \mathrm{E}$ & $1.15 \mathrm{G}$ \\
\hline Bank of Maharashtra & $0.92 \mathrm{~F}$ & $0.84 \mathrm{P}$ & $0.6 \mathrm{P}$ & $0.73 \mathrm{P}$ & $0.67 \mathrm{P}$ & $0.48 \mathrm{P}$ \\
\hline Canara bank & $1.07 \mathrm{G}$ & $1.09 \mathrm{G}$ & $1.25 \mathrm{E}$ & $1.39 \mathrm{E}$ & $1.17 \mathrm{G}$ & $1.58 \mathrm{E}$ \\
\hline Central Bank of India & $0.89 \mathrm{~F}$ & $0.79 \mathrm{P}$ & $0.51 \mathrm{P}$ & $0.74 \mathrm{P}$ & $0.52 \mathrm{P}$ & $0.41 \mathrm{P}$ \\
\hline Corporation bank & $1.37 \mathrm{E}$ & $1.4 \mathrm{E}$ & $1.79 \mathrm{E}$ & $1.34 \mathrm{E}$ & $1.39 \mathrm{E}$ & $1.75 \mathrm{E}$ \\
\hline Dena bank & $1.03 \mathrm{G}$ & $0.99 \mathrm{~F}$ & $0.81 \mathrm{~F}$ & $0.71 \mathrm{P}$ & $0.69 \mathrm{P}$ & $0.55 \mathrm{P}$ \\
\hline Indian bank & $0.87 \mathrm{P}$ & $0.82 \mathrm{P}$ & $1.22 \mathrm{G}$ & $0.89 \mathrm{~F}$ & $0.84 \mathrm{~F}$ & $1.2 \mathrm{G}$ \\
\hline Indian Overseas bank & $0.95 \mathrm{~F}$ & $0.96 \mathrm{~F}$ & $1 \mathrm{G}$ & $1.05 \mathrm{G}$ & $1.07 \mathrm{G}$ & $1.12 \mathrm{G}$ \\
\hline Oriental Bank of Commerce & $1.54 \mathrm{E}$ & $1.5 \mathrm{E}$ & $1.63 \mathrm{E}$ & $1.39 \mathrm{E}$ & $1.38 \mathrm{E}$ & $1.5 \mathrm{E}$ \\
\hline Punjab \& Sind bank & $0.98 \mathrm{~F}$ & $0.93 \mathrm{~F}$ & $0.77 \mathrm{P}$ & $0.79 \mathrm{P}$ & $0.74 \mathrm{P}$ & $0.59 \mathrm{P}$ \\
\hline Punjab National Bank & $0.87 \mathrm{P}$ & $0.94 \mathrm{~F}$ & $1.16 \mathrm{G}$ & $0.86 \mathrm{~F}$ & $0.93 \mathrm{~F}$ & $1.1 \mathrm{G}$ \\
\hline Syndicate bank & $0.98 \mathrm{~F}$ & $1 \mathrm{G}$ & $0.78 \mathrm{P}$ & $0.92 \mathrm{~F}$ & $0.95 \mathrm{~F}$ & $0.74 \mathrm{P}$ \\
\hline UCO bank & $0.99 \mathrm{~F}$ & $0.97 \mathrm{~F}$ & $0.61 \mathrm{P}$ & $0.96 \mathrm{~F}$ & $0.95 \mathrm{~F}$ & $0.57 \mathrm{P}$ \\
\hline Union Bank of India & $1.16 \mathrm{E}$ & $1.19 \mathrm{E}$ & $1.26 \mathrm{E}$ & $1.05 \mathrm{G}$ & $1.1 \mathrm{G}$ & $1.12 \mathrm{G}$ \\
\hline United Bank of India & $0.84 \mathrm{P}$ & $0.72 \mathrm{P}$ & $0.55 \mathrm{P}$ & $0.75 \mathrm{P}$ & $0.64 \mathrm{P}$ & $0.51 \mathrm{P}$ \\
\hline Vijaya bank & $1.11 \mathrm{G}$ & $1.03 \mathrm{G}$ & $0.94 \mathrm{~F}$ & $0.98 \mathrm{~F}$ & $0.92 \mathrm{~F}$ & $0.8 \mathrm{~F}$ \\
\hline State Bank of India & $0.84 \mathrm{P}$ & $0.88 \mathrm{~F}$ & $0.96 \mathrm{~F}$ & $1.26 \mathrm{E}$ & $1.31 \mathrm{E}$ & $1.3 \mathrm{E}$ \\
\hline Bank of Bikaner \& Jaipur & $0.79 \mathrm{P}$ & $0.84 \mathrm{P}$ & $0.8 \mathrm{~F}$ & $0.88 \mathrm{~F}$ & $0.95 \mathrm{~F}$ & $0.89 \mathrm{~F}$ \\
\hline State bank of Hyderabad & $1.12 \mathrm{G}$ & $1.1 \mathrm{G}$ & $1.23 \mathrm{E}$ & $1.11 \mathrm{G}$ & $1.1 \mathrm{G}$ & $1.24 \mathrm{G}$ \\
\hline State bank of Indore & $0.93 \mathrm{~F}$ & $1 \mathrm{G}$ & $1 \mathrm{G}$ & $1.05 \mathrm{G}$ & $1.14 \mathrm{G}$ & $1.13 \mathrm{G}$ \\
\hline State bank of Mysore & $0.76 \mathrm{P}$ & $0.83 \mathrm{P}$ & $0.84 \mathrm{~F}$ & $0.89 \mathrm{~F}$ & $0.99 \mathrm{~F}$ & $0.99 \mathrm{~F}$ \\
\hline State bank of Patiala & $1.12 \mathrm{G}$ & $1.17 \mathrm{E}$ & $1.09 \mathrm{G}$ & $1.26 \mathrm{E}$ & $1.34 \mathrm{E}$ & $1.24 \mathrm{G}$ \\
\hline State bank of Saurashtra & $0.56 \mathrm{P}$ & $0.52 \mathrm{P}$ & $0.36 \mathrm{P}$ & $0.75 \mathrm{P}$ & $0.7 \mathrm{P}$ & $0.49 \mathrm{P}$ \\
\hline State bank of Tranvancore & $0.9 \mathrm{~F}$ & $0.98 \mathrm{~F}$ & $1.01 \mathrm{G}$ & $1.17 \mathrm{E}$ & $1.29 \mathrm{E}$ & $1.3 \mathrm{E}$ \\
\hline Mean & 1 & 1 & 1 & 1 & 1 & 1 \\
\hline SD & 0.19 & 0.2 & 0.34 & 0.22 & 0.25 & 0.37 \\
\hline
\end{tabular}

Source: Calculated

The maximum employee productivity indices were revealed by Oriental bank of Commerce (1.54) in deposit per employee, Oriental bank of Commerce (1.5) in advances per employee and Corporation bank (1.79) in net profit per employee. On the other hand least productivity indices were revealed by State bank of Mysore (0.76) in deposit per employee, united bank of India (0.72) in advances per employee and Central bank of India (0.51) in net profit per employee. In branch productivity analysis, maximum productivity indices were revealed by Oriental bank of commerce (1.39) in deposits per branch, Corporation bank (1.39) in advances per branch and Corporation bank (1.75) in net profit per branch. On the other hand, least performance was revealed by Dena bank (0.71) in deposits per branch, central bank of India (0.52) in advances per branch and central bank of India (0.41) in net profit per branch.

\section{Conclusions}

In deposit per employee Bank of Baroda, Corporation bank, Oriental bank of Commerce, Union bank of India remain in excellent category in both periods except Bank of India, on the other hand State bank of Bikaner and Jaipur, State bank of Indore, state bank of Mysore and state bank of Saurashtra remain in poor category in addition Indian bank, PNB, United bank of India and SBI fall in this category except Syndicate bank and UCO bank.

Advances per employee shows that banks which are excellent maintained their excellence performance in both periods except SBOP, in addition Union bank of India also come under this category on the other hand, Bank of Maharashtra, Central bank of India, Indian bank, United bank of India, State bank of Bikaner and Jaipur, state bank of 
Mysore, state bank of Saurashtra remain poor while Syndicate bank, UCO bank and Vijaya bank improved in their performance.

Net profit per employee indicates improved performance of Andhra bank, Allahabad bank, Indian bank; Indian Oversea bank, Dena bank and Vijaya bank fall in excellent category and bank set of poor category remain at their previous level.

In deposit per branch SBOP, State bank of Tranvancore come under excellent category during 2001-10 period while Indian oversea bank slips to good category on the other hand, UCO bank, State bank of Indore State bank of Bikaner and Jaipur, state bank of Mysore shows improvement however, Punjab and Sind bank and state bank of Saurashtra fall in poor category.

Advances per branch shows mixed trend 2 new banks come in excellent category (SBOP and State bank of Tranvancore) while 2 banks (Canara bank and Indian Oversea bank) skips from this category on the other hand, Andhra bank, UCO bank and Vijaya bank has shown improved performance from poor category however, Punjab and Sind bank and state bank of Saurashtra shifted to poor category.

Net profit per branch depict that PNB, Union bank of India, State bank of Hyderabad, State bank of Indore, SBOP, United bank of India and State bank of Bikaner and Jaipur slip from excellent category, while Allahabad bank, Indian bank, Indian Oversea bank and Vijaya bank improve. On the other hand Dena bank, united bank of India and state bank of Saurashtra fall in poor category.

\section{Policy Implications}

To reduce overstaffing in public sector banks although efforts made to reduce the number of staff employed through VRS but they need to improve further. They should have a strategic tie up with the rural regional banks for reaching the far-fetched areas instead of opening branches themselves in the areas which cannot provide them the break even business.

They should embrace latest technology such as Mobile banking (M-banking) has the potential to unlock a large untapped market. Public sector banks have a unique advantage over their competition in terms of their branch network and the large customer base. But extending branch networks is often too expensive instead of opening new branches banks can significantly improve its services through adopting new techniques M-banking and able to provide 24 x 7 Banking (anywhere, anytime banking).

The face of banking is changing rapidly. Competition is going to be tough and with financial liberalization Indian public sector banks will have to benchmark themselves against the best in the world. These days introducing internationally followed best practices and observing universally acceptable standards and codes is necessary for strengthening the domestic financial architecture. This includes best practices in the area of corporate governance along with full transparency in disclosures.

For a strong and resilient banking and financial system, therefore, banks need to go beyond peripheral issues and tackle significant issues like improvements in profitability, maintain management efficiency level and technology and exploring available cost-effective solutions. 


\section{Appendix A}

\section{Deposit per Employee}

(Rs.in lakh)

\begin{tabular}{|c|c|c|c|c|c|c|c|c|c|c|c|c|c|}
\hline Name of Banks & 1991-92 & $1992-93$ & 1993-94 & $1994-95$ & $1995-96$ & $1996-97$ & $1997-98$ & $1998-99$ & 1999-00 & $2000-01$ & Mean & SD & $\mathrm{CV}$ \\
\hline ALLA & 29.89 & 33.82 & 36.95 & 40.17 & 44.2 & 50.55 & 59.9 & 69.41 & 79.74 & 95.7 & 54 & 21.7 & 40.23 \\
\hline AND & 24.74 & 26.86 & 30.02 & 32.47 & 39.16 & 47.07 & 53.03 & 70.67 & 98.73 & 143 & 56.6 & 38 & 67.24 \\
\hline $\mathrm{BOB}$ & 42.47 & 46.91 & 53.89 & 58.05 & 61.83 & 70.3 & 85.18 & 96.59 & 109 & 117 & 74.1 & 26.3 & 35.51 \\
\hline BOI & 36.56 & 36.68 & 40.3 & 45.01 & 50.93 & 59.99 & 74.91 & 83.76 & 91.07 & 117 & 63.7 & 27.3 & 42.84 \\
\hline BOM & 19.33 & 20.76 & 23.68 & 30.52 & 34.64 & 43.7 & 55.04 & 66.82 & 83.28 & 120 & 49.7 & 32.3 & 64.97 \\
\hline CAN & 27.65 & 31.69 & 37.62 & 42.15 & 48.56 & 57.89 & 69.55 & 76.15 & 86.7 & 122 & 60 & 29.4 & 48.92 \\
\hline CBI & 25.23 & 26.67 & 29.25 & 34.25 & 38.61 & 45.76 & 53.06 & 62.64 & 74.33 & 87.5 & 47.7 & 21.3 & 44.7 \\
\hline CORP & 25.93 & 31.56 & 45 & 66.75 & 62.08 & 71.15 & 97.26 & 123.8 & 134.9 & 161 & 81.9 & 45.7 & 55.78 \\
\hline DENA & 20.77 & 25.11 & 29.76 & 35.52 & 40.57 & 50.36 & 66.95 & 79.26 & 92.19 & 133 & 57.4 & 35.7 & 62.25 \\
\hline IND & 34.85 & 44.48 & 44.55 & 47.65 & 48.9 & 52.55 & 57.13 & 63.97 & 72.39 & 94.7 & 56.1 & 17.2 & 30.73 \\
\hline IOB & 27.15 & 32.69 & 37.46 & 43.49 & 51.21 & 55.76 & 68.19 & 77.34 & 86.19 & 105 & 58.5 & 25.4 & 43.37 \\
\hline $\mathrm{OBC}$ & 33.15 & 37.64 & 44.06 & 53.96 & 66.35 & 74.04 & 91.71 & 116.3 & 153.5 & 182 & 85.2 & 50.7 & 59.43 \\
\hline PSB & 23 & 27.16 & 31.97 & 42.36 & 47.09 & 51.82 & 62.55 & 77.89 & 87.04 & 118 & 56.9 & 30 & 52.76 \\
\hline PNB & 27.23 & 30.41 & 30.16 & 34.24 & 38.06 & 45.56 & 52.81 & 62.06 & 73.35 & 96.3 & 49 & 22.4 & 45.8 \\
\hline SYND & 20.59 & 23.07 & 26.44 & 31.15 & 34.06 & 40.5 & 46.37 & 55.71 & 68.41 & 81.7 & 42.8 & 20.3 & 47.48 \\
\hline UCO Bank & 27.07 & 28.22 & 26.7 & 29.65 & 33.45 & 37.57 & 43.95 & 50.65 & 58.8 & 73.8 & 41 & 15.8 & 38.63 \\
\hline UNI & 24.23 & 28.38 & 36.03 & 47.07 & 54.29 & 64.7 & 74.61 & 91.25 & 102.4 & 124 & 64.7 & 33.3 & 51.46 \\
\hline UTD & 23.81 & 27.09 & 30.83 & 35.65 & 39.06 & 46.39 & 54.62 & 66.72 & 78.76 & 92.6 & 49.5 & 23.2 & 46.87 \\
\hline VIJ & 20.01 & 24.03 & 30.67 & 41.6 & 41.88 & 47.85 & 58.11 & 68.53 & 80.93 & 93.8 & 50.7 & 24.4 & 48.12 \\
\hline SBI & 26.86 & 29.32 & 33.35 & 36.69 & 41.38 & 46.87 & 54.7 & 71.17 & 84.32 & 113 & 53.8 & 27.8 & 51.79 \\
\hline SBBJ & 17.5 & 19.98 & 23.4 & 26.39 & 30.94 & 35.8 & 43.37 & 51.71 & 61.4 & 77.1 & 38.8 & 19.5 & 50.41 \\
\hline $\mathrm{SBH}$ & 23.38 & 28.23 & 35.07 & 39.94 & 43.44 & 51.27 & 60.61 & 72.64 & 84.99 & 112 & 55.1 & 27.8 & 50.41 \\
\hline INDO & 19.13 & 22.33 & 26.51 & 30.7 & 36.51 & 41.07 & 49.32 & 58.74 & 73.58 & 102 & 46 & 26 & 56.56 \\
\hline SBM & 17.49 & 21.8 & 24.87 & 30.34 & 35.07 & 39.37 & 42.52 & 48.74 & 58.08 & 76.3 & 39.5 & 18 & 45.57 \\
\hline SBP & 22.14 & 26.11 & 33.75 & 38.95 & 45.34 & 51.62 & 59.03 & 67.5 & 77.34 & 96.9 & 51.9 & 23.7 & 45.71 \\
\hline SBS & 17.35 & 21.87 & 25.69 & 32.51 & 39.13 & 46.16 & 49.98 & 58.62 & 70.99 & 89 & 45.1 & 22.8 & 50.56 \\
\hline SBT & 22.32 & 25.95 & 31.73 & 37.63 & 42.19 & 49.76 & 57.23 & 65.42 & 78.61 & 95.1 & 50.6 & 23.6 & 46.73 \\
\hline
\end{tabular}




\begin{tabular}{|c|c|c|c|c|c|c|c|c|c|c|c|c|c|}
\hline Name of banks & 2001-02 & $2002-03$ & 2003-04 & $2004-05$ & 2005-06 & 2006-07 & 2007-08 & 2008-09 & $2009-10$ & $2010-11$ & Mean & $\mathrm{SD}$ & $\mathrm{CV}$ \\
\hline ALLA & 114 & 130 & 163 & 211 & 253 & 292 & 357 & 415 & 506 & 621 & 306 & 168 & 54.82 \\
\hline AND & 144 & 162 & 175 & 210 & 242 & 300 & 369 & 417 & 544 & 654 & 322 & 173 & 53.69 \\
\hline $\mathrm{BOB}$ & 159 & 165 & 183 & 209 & 242 & 328 & 405 & 528 & 619 & 776 & 361 & 215 & 59.59 \\
\hline BOI & 138 & 149 & 167 & 187 & 223 & 289 & 369 & 472 & 586 & 751 & 333 & 210 & 62.92 \\
\hline $\mathrm{BOM}$ & 135 & 158 & 187 & 204 & 191 & 244 & 307 & 383 & 463 & 482 & 276 & 127 & 46.08 \\
\hline CAN & 134 & 152 & 181 & 204 & 249 & 307 & 340 & 424 & 541 & 677 & 321 & 179 & 55.77 \\
\hline CBI & 119 & 130 & 144 & 159 & 166 & 212 & 294 & 400 & 504 & 527 & 266 & 158 & 59.35 \\
\hline CORP & 185 & 202 & 216 & 267 & 290 & 357 & 461 & 594 & 706 & 842 & 412 & 231 & 56.02 \\
\hline DENA & 144 & 156 & 177 & 197 & 233 & 274 & 341 & 436 & 488 & 645 & 309 & 167 & 53.91 \\
\hline IND & 108 & 123 & 140 & 161 & 191 & 225 & 297 & 363 & 449 & 548 & 260 & 150 & 57.65 \\
\hline IOB & 129 & 150 & 170 & 182 & 209 & 288 & 341 & 392 & 412 & 567 & 284 & 142 & 50.13 \\
\hline OBC & 210 & 221 & 263 & 329 & 335 & 434 & 526 & 671 & 783 & 837 & 461 & 233 & 50.51 \\
\hline PSB & 126 & 135 & 140 & 147 & 177 & 207 & 276 & 399 & 595 & 737 & 294 & 216 & 73.37 \\
\hline PNB & 111 & 129 & 149 & 177 & 206 & 137 & 297 & 383 & 467 & 549 & 261 & 156 & 59.96 \\
\hline SYND & 112 & 113 & 159 & 186 & 218 & 323 & 386 & 462 & 458 & 516 & 293 & 155 & 52.72 \\
\hline UCO Bank & 105 & 125 & 156 & 199 & 223 & 262 & 335 & 422 & 524 & 630 & 298 & 177 & 59.41 \\
\hline UNI & 154 & 174 & 197 & 228 & 275 & 309 & 382 & 478 & 578 & 687 & 346 & 182 & 52.54 \\
\hline UTD & 109 & 119 & 127 & 145 & 169 & 221 & 293 & 361 & 446 & 517 & 251 & 147 & 58.62 \\
\hline VIJ & 124 & 146 & 181 & 223 & 254 & 349 & 419 & 455 & 536 & 642 & 333 & 176 & 52.92 \\
\hline SBI & 129 & 142 & 154 & 179 & 191 & 235 & 300 & 360 & 401 & 419 & 251 & 111 & 44.14 \\
\hline SBBJ & 87.7 & 101 & 120 & 148 & 179 & 242 & 299 & 343 & 373 & 471 & 236 & 131 & 55.36 \\
\hline SBH & 129 & 154 & 183 & 221 & 260 & 322 & 391 & 497 & 537 & 656 & 335 & 179 & 53.61 \\
\hline INDO & 121 & 141 & 159 & 214 & 251 & 307 & 394 & 451 & 481 & & 280 & 136 & 48.52 \\
\hline SBM & 86.4 & 92.5 & 114 & 142 & 168 & 229 & 283 & 340 & 385 & 435 & 228 & 127 & 55.87 \\
\hline SBP & 118 & 153 & 193 & 229 & 298 & 346 & 435 & 528 & 520 & 542 & 336 & 162 & 48.35 \\
\hline SBS & 102 & 122 & 144 & 172 & 191 & 221 & 232 & & & & 169 & 49 & 28.93 \\
\hline SBT & 111 & 133 & 164 & 204 & 223 & 267 & 326 & 370 & 417 & 482 & 270 & 125 & 46.51 \\
\hline
\end{tabular}


Advances per Employee

(Rs.in lakh)

\begin{tabular}{|c|c|c|c|c|c|c|c|c|c|c|c|c|c|}
\hline Name of Banks & $1991-92$ & $1992-93$ & 1993-94 & $1994-95$ & $1995-96$ & 1996-97 & $1997-98$ & $1998-99$ & 1999-00 & 2000-01 & Mean & SD & $\mathrm{CV}$ \\
\hline ALLA & 15.44 & 17.39 & 16.26 & 18.44 & 20.97 & 21.63 & 25.32 & 31.26 & 37.24 & 45.61 & 24.96 & 10.06 & 40.3 \\
\hline AND & 12.2 & 12.24 & 11.74 & 14.73 & 16.93 & 19.3 & 22.07 & 30.63 & 38.17 & 58 & 23.6 & 14.87 & 62.99 \\
\hline $\mathrm{BOB}$ & 23.34 & 26.35 & 29.49 & 32.83 & 34.9 & 36.13 & 43.11 & 45.67 & 51.84 & 80.72 & 40.44 & 16.68 & 41.26 \\
\hline BOI & 23.93 & 21.71 & 20.55 & 22.44 & 28.86 & 34.41 & 41.93 & 44.28 & 48.13 & 73.03 & 35.93 & 16.49 & 45.91 \\
\hline $\mathrm{BOM}$ & 10.42 & 9.942 & 9.549 & 13.39 & 15.62 & 18.46 & 21.81 & 24.83 & 32.63 & 46.86 & 20.35 & 11.9 & 58.46 \\
\hline CAN & 15.54 & 15.48 & 15.71 & 20.4 & 24.23 & 26.54 & 30.76 & 35.45 & 42.53 & 57.67 & 28.43 & 13.7 & 48.2 \\
\hline CBI & 12.98 & 7.28 & 10.98 & 15.15 & 17.4 & 17.45 & 21.48 & 26.16 & 32.75 & 39.67 & 20.13 & 10.1 & 50.19 \\
\hline CORP & 11.17 & 13.65 & 15.55 & 22.48 & 26.44 & 32.15 & 44.75 & 61.74 & 73.46 & 84.18 & 38.56 & 26.29 & 68.2 \\
\hline DENA & 10.17 & 12.05 & 13.04 & 17.64 & 21.31 & 25.91 & 34.07 & 42.98 & 49.39 & 63.96 & 29.05 & 18.12 & 62.36 \\
\hline IND & 25.18 & 30 & 25.34 & 29.46 & 28.89 & 25.18 & 26.89 & 28.05 & 31.07 & 41.2 & 29.13 & 4.73 & 16.27 \\
\hline IOB & 17.84 & 18.48 & 18.51 & 22.71 & 26.34 & 25.32 & 30.57 & 35.71 & 41.02 & 50.35 & 28.69 & 10.81 & 37.69 \\
\hline $\mathrm{OBC}$ & 16.24 & 19.53 & 21.59 & 28.54 & 35.59 & 35.98 & 44.37 & 53.35 & 64.77 & 81.52 & 40.15 & 21.15 & 52.67 \\
\hline PSB & 11.67 & 12.43 & 13.47 & 19.57 & 22.35 & 22.67 & 26.19 & 33.63 & 39.29 & 51.38 & 25.26 & 12.85 & 50.85 \\
\hline PNB & 14.01 & 16.53 & 13.57 & 16.1 & 17.79 & 20.8 & 24.09 & 28.99 & 34.87 & 48.09 & 23.48 & 11.04 & 47 \\
\hline SYND & 10.46 & 11 & 10.41 & 11.69 & 14.45 & 15.8 & 19.19 & 26.05 & 35.3 & 42.71 & 19.71 & 11.39 & 57.79 \\
\hline UCO Bank & 18.24 & 16.4 & 13.14 & 14 & 14.57 & 14.6 & 17.05 & 19.39 & 24.44 & 34.58 & 18.64 & 6.503 & 34.88 \\
\hline UNI & 11.23 & 13.65 & 15.76 & 21.79 & 26.34 & 29.65 & 33.25 & 35.41 & 48.11 & 62.42 & 29.76 & 16.06 & 53.97 \\
\hline UTD & 12.47 & 12.87 & 11.15 & 12.48 & 12.67 & 13.57 & 15.29 & 17.67 & 21.41 & 28.75 & 15.83 & 5.47 & 34.59 \\
\hline VIJ & 11.27 & 11.66 & 13.04 & 16.7 & 17.09 & 17.35 & 22.81 & 26.64 & 32.73 & 42.46 & 21.17 & 10.14 & 47.88 \\
\hline SBI & 19.79 & 21.08 & 18.11 & 20.92 & 25.68 & 26.35 & 30.98 & 34.68 & 42.03 & 52.87 & 29.25 & 11.18 & 38.22 \\
\hline SBBJ & 10.43 & 11.69 & 12.12 & 13.82 & 16.25 & 19.98 & 24.33 & 25.66 & 29.78 & 38.59 & 20.26 & 9.22 & 45.54 \\
\hline SBH & 14.39 & 16.37 & 17.3 & 23.71 & 27.64 & 28.63 & 32.45 & 36.49 & 41.3 & 53.4 & 29.17 & 12.27 & 42.06 \\
\hline INDO & 12.48 & 13.81 & 15.37 & 18.67 & 21.77 & 23.59 & 27.84 & 30.92 & 41.03 & 52.25 & 25.77 & 12.75 & 49.48 \\
\hline SBM & 10.59 & 12.84 & 12.77 & 16.6 & 18.83 & 21.71 & 23.46 & 26.11 & 30.61 & 43 & 21.65 & 9.84 & 45.45 \\
\hline SBP & 14.02 & 15.89 & 16.57 & 20.38 & 24.12 & 27.04 & 31.32 & 36.72 & 43.87 & 57.21 & 28.72 & 13.88 & 48.33 \\
\hline SBS & 11.67 & 13.68 & 13.73 & 19.03 & 22.57 & 26.89 & 30.01 & 33.37 & 39.35 & 47.96 & 25.83 & 12.01 & 46.52 \\
\hline SBT & 12.2 & 15.34 & 17.86 & 24.56 & 26.05 & 28.17 & 30.66 & 32.13 & 39.61 & 52.56 & 27.91 & 11.97 & 42.9 \\
\hline
\end{tabular}




\begin{tabular}{|c|c|c|c|c|c|c|c|c|c|c|c|c|c|}
\hline Name of banks & 2001-02 & $2002-03$ & 2003-04 & 2004-05 & 2005-06 & 2006-07 & $2007-08$ & 2008-09 & 2009-10 & 2010-11 & Mean & $\mathrm{SD}$ & $\mathrm{CV}$ \\
\hline ALLA & 55.3 & 64.3 & 79.6 & 110 & 152 & 203 & 248 & 287 & 342 & 441 & 198 & 130 & 65.67 \\
\hline AND & 75.5 & 88.6 & 98.4 & 134 & 158 & 202 & 256 & 310 & 393 & 507 & 222 & 144 & 64.73 \\
\hline ВОВ & 86.5 & 87.7 & 89.4 & 112 & 155 & 220 & 285 & 393 & 449 & 581 & 246 & 176 & 71.6 \\
\hline BOI & 88.2 & 98.8 & 108 & 133 & 154 & 205 & 279 & 356 & 430 & 536 & 239 & 156 & 65.34 \\
\hline BOM & 58.4 & 67.7 & 82.8 & 92.5 & 117 & 165 & 215 & 252 & 295 & 338 & 168 & 101 & 60.03 \\
\hline CAN & 69.3 & 85.1 & 100 & 128 & 169 & 212 & 237 & 313 & 390 & 490 & 219 & 140 & 63.95 \\
\hline CBI & 53.7 & 5.73 & 58.6 & 71.2 & 93.4 & 133 & 195 & 261 & 328 & 381 & 158 & 128 & 80.88 \\
\hline CORP & 107 & 112 & 129 & 182 & 212 & 252 & 326 & 389 & 481 & 627 & 282 & 173 & 61.51 \\
\hline DENA & 70.3 & 79.9 & 91 & 111 & 140 & 181 & 231 & 292 & 337 & 450 & 198 & 127 & 64.19 \\
\hline IND & 48.9 & 55.7 & 64.8 & 85.2 & 105 & 139 & 194 & 257 & 316 & 390 & 166 & 120 & 72.25 \\
\hline IOB & 61.5 & 71.3 & 83.2 & 103 & 144 & 197 & 244 & 294 & 294 & 436 & 193 & 123 & 63.92 \\
\hline $\mathrm{OBC}$ & 104 & 116 & 145 & 174 & 224 & 300 & 369 & 467 & 544 & 577 & 302 & 178 & 59.11 \\
\hline PSB & 56.2 & 60 & 61.7 & 65.6 & 95.4 & 126 & 204 & 283 & 395 & 526 & 187 & 164 & 87.84 \\
\hline PNB & 59.4 & 68.2 & 80.3 & 104 & 129 & 169 & 213 & 282 & 349 & 425 & 188 & 127 & 67.63 \\
\hline SYND & 58.2 & 60.2 & 77.2 & 108 & 148 & 212 & 260 & 325 & 354 & 406 & 201 & 130 & 64.6 \\
\hline UCO Bank & 50.1 & 63.5 & 82.1 & 111 & 152 & 190 & 231 & 290 & 353 & 430 & 195 & 129 & 66.17 \\
\hline UNI & 82.8 & 99.3 & 115 & 148 & 198 & 227 & 274 & 333 & 406 & 512 & 239 & 142 & 59.42 \\
\hline UTD & 38 & 41.4 & 44.5 & 65.1 & 89.6 & 132 & 174 & 234 & 277 & 355 & 145 & 112 & 76.98 \\
\hline VIJ & 52.4 & 67.3 & 95 & 125 & 153 & 225 & 277 & 296 & 359 & 427 & 208 & 129 & 62.25 \\
\hline SBI & 57.7 & 65.9 & 76.3 & 98.5 & 132 & 182 & 233 & 263 & 315 & 339 & 176 & 106 & 60.12 \\
\hline SBBJ & 44.6 & 51.3 & 65.9 & 93.2 & 131 & 175 & 220 & 261 & 285 & 360 & 169 & 110 & 64.99 \\
\hline SBH & 62.5 & 72.2 & 89 & 119 & 159 & 218 & 280 & 347 & 390 & 479 & 222 & 147 & 66.14 \\
\hline INDO & 65.6 & 79.4 & 98 & 140 & 179 & 236 & 291 & 344 & 372 & & 200 & 116 & 57.65 \\
\hline SBM & 49.8 & 54 & 65.1 & 91.8 & 121 & 171 & 216 & 265 & 292 & 343 & 167 & 107 & 64.19 \\
\hline SBP & 73.3 & 92.2 & 112 & 132 & 195 & 254 & 326 & 384 & 373 & 410 & 235 & 131 & 55.62 \\
\hline SBS & 55.3 & 62.8 & 70.9 & 91.7 & 116 & 155 & 175 & & & & 104 & 46.9 & 45.1 \\
\hline SBT & 61.3 & 76.4 & 92.7 & 126 & 162 & 214 & 260 & 287 & 315 & 381 & 197 & 111 & 56.1 \\
\hline
\end{tabular}


Net profit per employee

(Rs.in lakh)

\begin{tabular}{|c|c|c|c|c|c|c|c|c|c|c|c|c|c|}
\hline Name of Banks & $1991-92$ & $1992-93$ & $1993-94$ & 1994-95 & $1995-96$ & $1996-97$ & $1997-98$ & 1998-99 & $1999-00$ & $2000-01$ & Mean & SD & $\mathrm{CV}$ \\
\hline ALLA & 0.13 & -0.47 & -1.61 & -0.33 & 0.03 & 0.28 & 0.57 & 0.6 & 0.31 & 0.19 & -0 & 0.65 & -2218 \\
\hline AND & 0.05 & -0.9 & -0.98 & -0.27 & 0.07 & 0.24 & 0.5 & 0.61 & 0.83 & 0.95 & 0.11 & 0.67 & 613 \\
\hline ВOB & 0.21 & 0.02 & 0.15 & 0.39 & 0.45 & 0.61 & 1 & 0.91 & 1.07 & 0.59 & 0.54 & 0.36 & 67.5 \\
\hline BOI & 0.11 & -0.62 & -2.05 & 0.09 & 0.51 & 0.68 & 0.69 & 0.38 & 0.33 & 0.57 & 0.07 & 0.84 & 1214 \\
\hline BOM & 0.02 & -1.06 & -1.57 & -0.23 & 0.08 & 0.28 & 0.34 & 0.32 & 0.56 & 0.32 & -0.1 & 0.69 & -724 \\
\hline CAN & 0.3 & 0.05 & 0.23 & 0.38 & 0.47 & 0.27 & 0.37 & 0.41 & 0.43 & 0.59 & 0.35 & 0.15 & 42.2 \\
\hline CBI & 0.06 & -0.74 & -1.3 & -0.16 & -0.1 & 0.3 & 0.35 & 0.3 & 0.31 & 0.1 & -0.1 & 0.54 & -579 \\
\hline CORP & 0.06 & 0.04 & 0.3 & 0.79 & 1.14 & 1.33 & 1.74 & 1.89 & 2.2 & 2.54 & 1.2 & 0.89 & 74.4 \\
\hline DENA & 0.05 & -0.55 & -0.42 & 0.18 & 0.33 & 0.47 & 0.69 & 0.74 & 0.44 & -2.4 & -0 & 0.94 & -1894 \\
\hline IND & 0.14 & 0.03 & -1.46 & 0.05 & -4.9 & -1.4 & -1.1 & -2.9 & 1.62 & -1.2 & -1.1 & 1.81 & -162 \\
\hline IOB & 0.03 & -2.59 & -1.22 & 0.03 & 0.01 & 0.37 & 0.4 & 0.2 & 0.14 & 0.45 & -0.2 & 0.96 & -439 \\
\hline $\mathrm{OBC}$ & 0.25 & 0.18 & 0.27 & 0.92 & 1.32 & 1.33 & 1.47 & 1.59 & 1.94 & 1.49 & 1.08 & 0.63 & 58.9 \\
\hline PSB & 0.01 & -1.56 & -1.4 & -0.06 & -1.1 & 0.16 & 0.53 & 0.46 & 0.51 & 0.13 & -0.2 & 0.8 & -354 \\
\hline PNB & 0.19 & 0.06 & 0.1 & 0.12 & -0.1 & 0.35 & 0.72 & 0.57 & 0.63 & 0.8 & 0.34 & 0.32 & 93.8 \\
\hline SYND & 0.01 & -1.74 & -0.78 & -0.24 & 0.05 & 0.18 & 0.23 & 0.4 & 0.62 & 0.76 & -0.1 & 0.74 & -1457 \\
\hline UCO Bank & -0.06 & -1.25 & -1.57 & -0.24 & -0.7 & -0.5 & -0.3 & -0.2 & 0.12 & 0.11 & -0.5 & 0.57 & -123 \\
\hline UNI & 0.09 & 0.03 & 0.15 & 0.32 & 0.24 & 0.7 & 0.81 & 0.52 & 0.33 & 0.55 & 0.38 & 0.26 & 69.6 \\
\hline UTD & 0.03 & -1.23 & 2.72 & 0.87 & 1.04 & -0.5 & 0.05 & 0.07 & 0.15 & 0.1 & 0.33 & 1.05 & 321 \\
\hline VIJ & 0.01 & -0.71 & 0.03 & 0.23 & -1.8 & 0.13 & 0.16 & 0.21 & 0.37 & 0.53 & -0.1 & 0.67 & -848 \\
\hline SBI & 0.08 & 0.09 & 0.12 & 0.31 & 0.36 & 0.57 & 0.78 & 0.43 & 0.88 & 0.75 & 0.44 & 0.3 & 68.1 \\
\hline SBBJ & 0.07 & 0.08 & 0.05 & 0.05 & 0.17 & 0.27 & 0.6 & 0.61 & 0.81 & 0.79 & 0.35 & 0.32 & 91 \\
\hline SBH & 0.1 & 0.12 & 0.17 & 0.32 & 0.36 & 0.37 & 0.68 & 0.76 & 0.87 & 1.13 & 0.49 & 0.35 & 72.2 \\
\hline INDO & 0.05 & 0.04 & 0.06 & 0.13 & 0.18 & 0.25 & 0.41 & 0.45 & 0.65 & 0.98 & 0.32 & 0.31 & 95.6 \\
\hline SBM & 0.04 & 0.04 & 0.02 & 0.03 & 0.24 & 0.36 & 0.45 & 0.29 & 0.42 & 0.26 & 0.22 & 0.17 & 79.8 \\
\hline SBP & 0.24 & 0.15 & 0.22 & 0.24 & 0.38 & 0.42 & 1.09 & 0.77 & 0.99 & 1.35 & 0.59 & 0.43 & 73.5 \\
\hline SBS & 0.07 & 0.06 & 0.07 & 0.17 & -2.9 & 0.89 & 0.98 & 0.31 & 1.06 & 0.18 & 0.09 & 1.11 & 1183 \\
\hline
\end{tabular}




\begin{tabular}{|c|c|c|c|c|c|c|c|c|c|c|c|c|c|}
\hline SBT & 0.04 & 0.06 & 0.07 & 0.16 & 0.2 & 0.31 & 0.48 & 0.33 & 0.51 & 0.8 & 0.3 & 0.24 & 81.8 \\
\hline Name of banks & 2001-02 & $2002-03$ & 2003-04 & 2004-05 & 2005-06 & 2006-07 & 2007-08 & 2008-09 & 2009-10 & $2010-11$ & Mean & SD & $\mathrm{CV}$ \\
\hline ALLA & 0.4 & 0.85 & 2.4 & 2.81 & 3.69 & 3.68 & 4.85 & 3.76 & 5.76 & 6.7 & 3.49 & 1.99 & 56.97 \\
\hline AND & 1.58 & 3.1 & 3.54 & 3.97 & 3.46 & 3.89 & 4.3 & 4.58 & 7.32 & 9 & 4.47 & 2.14 & 47.93 \\
\hline ВOB & 1.4 & 1.92 & 2.43 & 1.74 & 2.13 & 2.7 & 3.83 & 6.11 & 7.85 & 11 & 4.11 & 3.2 & 77.78 \\
\hline BOI & 1.16 & 1.97 & 2.37 & 0.81 & 1.66 & 2.71 & 4.95 & 7.49 & 4.44 & 6.2 & 3.38 & 2.27 & 67.25 \\
\hline BOM & 1.03 & 1.58 & 2.15 & 1.25 & 0.36 & 1.96 & 2.42 & 2.75 & 3.21 & 2.38 & 1.91 & 0.86 & 45.07 \\
\hline CAN & 1.55 & 2.14 & 2.81 & 2.34 & 2.86 & 3.06 & 3.46 & 4.7 & 6.97 & 9.76 & 3.97 & 2.55 & 64.3 \\
\hline CBI & 0.41 & 0.78 & 1.59 & 0.93 & 0.64 & 1.28 & 1.47 & 1.74 & 3.29 & 3.95 & 1.61 & 1.15 & 71.77 \\
\hline CORP & 3.01 & 3.88 & 4.7 & 3.94 & 3.92 & 4.51 & 6.12 & 7.16 & 8.9 & 10.9 & 5.71 & 2.56 & 44.88 \\
\hline DENA & 0.11 & 1.08 & 2.23 & 0.6 & 0.72 & 1.99 & 3.61 & 4.28 & 4.86 & 6.15 & 2.56 & 2.06 & 80.3 \\
\hline IND & 0.15 & 0.86 & 1.86 & 1.89 & 2.36 & 3.64 & 4.91 & 6.23 & 7.92 & 8.88 & 3.87 & 3.01 & 77.7 \\
\hline IOB & 0.93 & 1.7 & 2.1 & 2.67 & 3.24 & 4.23 & 4.86 & 5.2 & 2.63 & 4.15 & 3.17 & 1.41 & 44.44 \\
\hline $\mathrm{OBC}$ & 2.36 & 3.38 & 5.05 & 5.22 & 3.72 & 3.94 & 5.68 & 6.08 & 7.39 & 9.04 & 5.19 & 1.99 & 38.39 \\
\hline PSB & 0.23 & 0.05 & 0.09 & -0.74 & 1.14 & 2.34 & 4.24 & 4.96 & 6.16 & 6 & 2.45 & 2.67 & 109 \\
\hline PNB & 0.97 & 1.43 & 1.88 & 2.42 & 2.48 & 2.69 & 3.66 & 5.64 & 7.31 & 8.35 & 3.68 & 2.55 & 69.22 \\
\hline SYND & 0.98 & 1.27 & 1.62 & 1.62 & 2.18 & 2.94 & 3.44 & 3.64 & 3.18 & 3.99 & 2.49 & 1.08 & 43.5 \\
\hline UCO Bank & 0.64 & 0.83 & 1.73 & 1.39 & 0.8 & 1.28 & 1.73 & 2.35 & 4.33 & 4.19 & 1.93 & 1.33 & 69.15 \\
\hline UNI & 1.22 & 2.15 & 2.78 & 2.65 & 2.5 & 3.07 & 5.11 & 5.95 & 7.05 & 7.5 & 4 & 2.22 & 55.43 \\
\hline UTD & 0.66 & 1.72 & 1.76 & 1.72 & 1.18 & 1.59 & 1.99 & 1.22 & 2.11 & 3.48 & 1.74 & 0.74 & 42.71 \\
\hline VIJ & 1.11 & 1.68 & 3.54 & 3.31 & 1.16 & 3.08 & 3.16 & 2.19 & 4.39 & 6.3 & 2.99 & 1.58 & 52.98 \\
\hline SBI & 1.16 & 1.49 & 1.78 & 2.09 & 2.22 & 2.45 & 3.75 & 4.43 & 4.58 & 6.44 & 3.04 & 1.69 & 55.75 \\
\hline SBBJ & 1.24 & 1.54 & 2.31 & 1.6 & 1.2 & 2.6 & 2.76 & 3.53 & 3.68 & 5 & 2.55 & 1.24 & 48.69 \\
\hline SBH & 1.68 & 2.25 & 2.87 & 1.91 & 3.26 & 3.92 & 4.35 & 4.9 & 6.05 & 7.89 & 3.91 & 1.97 & 50.3 \\
\hline INDO & 1.91 & 3.07 & 3.46 & 2.06 & 2.09 & 2.91 & 3.73 & 4.44 & 4.83 & & 3.17 & 1.05 & 33.12 \\
\hline SBM & 0.67 & 1.19 & 1.82 & 2.16 & 2.22 & 2.6 & 3.28 & 3.48 & 4.41 & 5 & 2.68 & 1.37 & 51.04 \\
\hline SBP & 1.97 & 2.76 & 3.69 & 2.48 & 2.67 & 3.24 & 3.7 & 4.68 & 4.44 & 5.2 & 3.48 & 1.05 & 30.16 \\
\hline SBS & 1.1 & 1.25 & 2.4 & 0.56 & 0.83 & 1.22 & 0.74 & & & & 1.16 & 0.6 & 52.18 \\
\hline SBT & 1 & 1.42 & 2.04 & 2.09 & 2.22 & 2.81 & 3.56 & 5.35 & 5.61 & 6 & 3.21 & 1.83 & 56.97 \\
\hline
\end{tabular}


Deposit per branch

(Rs.in lakh)

\begin{tabular}{|c|c|c|c|c|c|c|c|c|c|c|c|c|c|}
\hline Name of banks & $1991-92$ & $1992-93$ & 1993-94 & $1994-95$ & 1995-96 & $1996-97$ & $1997-98$ & 1998-99 & 1999-00 & $2000-01$ & Mean & SD & $\mathrm{CV}$ \\
\hline ALLA & 368.7 & 426.6 & 458.4 & 497.6 & 545.4 & 619.48 & 722.2 & 823.3 & 932 & 1057 & 645.1 & 231.2 & 35.84 \\
\hline AND & 406 & 436.7 & 509.9 & 547.6 & 610.1 & 728.03 & 806.6 & 1038 & 1416 & 1793 & 829.2 & 458.3 & 55.27 \\
\hline BOB & 805.8 & 871.3 & 950.1 & 1070 & 1146 & 1290.3 & 1551 & 1734 & 1935 & 2026 & 1338 & 446.5 & 33.37 \\
\hline BOI & 817.4 & 819.7 & 896.2 & 1011 & 1122 & 1291.8 & 1577 & 1767 & 1886 & 2039 & 1323 & 462 & 34.93 \\
\hline BOM & 300.2 & 341.9 & 396.8 & 470.2 & 524.7 & 642.11 & 786.1 & 930.1 & 1122 & 1397 & 691.1 & 363.6 & 52.61 \\
\hline CAN & 702.8 & 804 & 949.7 & 1052 & 1197 & 1390.1 & 1646 & 1764 & 2003 & 2456 & 1396 & 565.4 & 40.49 \\
\hline CBI & 433.3 & 454.4 & 521.5 & 573.8 & 640.5 & 746.71 & 854 & 990.9 & 1156 & 1335 & 770.7 & 308.3 & 40 \\
\hline CORP & 532.2 & 637.1 & 874.3 & 1247 & 1145 & 1316.2 & 1610 & 2042 & 2204 & 2540 & 1415 & 675.5 & 47.75 \\
\hline DENA & 310.4 & 371 & 439.3 & 511.4 & 571.1 & 687.75 & 875 & 1012 & 1136 & 1240 & 715.3 & 331.2 & 46.3 \\
\hline IND & 655.9 & 835.2 & 846.3 & 884.7 & 904.6 & 963.62 & 1032 & 1142 & 1278 & 1524 & 1007 & 250.7 & 24.91 \\
\hline IOB & 605.8 & 726.8 & 813 & 944.3 & 1077 & 1165.1 & 1401 & 1563 & 1707 & 1910 & 1191 & 439.8 & 36.92 \\
\hline $\mathrm{OBC}$ & 675.2 & 773.4 & 898.6 & 1080 & 1243 & 1331.7 & 1553 & 1869 & 2415 & 2648 & 1449 & 675.7 & 46.64 \\
\hline PSB & 393 & 442.7 & 588.5 & 763.7 & 839.6 & 906.25 & 1070 & 1308 & 1434 & 1596 & 934.2 & 413.5 & 44.26 \\
\hline PNB & 531.5 & 590.1 & 596.6 & 667.8 & 726.4 & 818.22 & 927.3 & 1067 & 1232 & 1447 & 860.4 & 305 & 35.45 \\
\hline SYND & 489.7 & 569 & 649 & 751.1 & 800.4 & 927.75 & 1101 & 1192 & 1390 & 1448 & 931.9 & 338.3 & 36.3 \\
\hline UCO Bank & 536.7 & 558.4 & 517.7 & 576 & 635 & 699.61 & 802.1 & 905.9 & 1040 & 1251 & 752.3 & 246 & 32.7 \\
\hline UNI & 429.8 & 498.6 & 622.7 & 806.9 & 892.8 & 985.47 & 1105 & 1328 & 1456 & 1699 & 982.4 & 419.1 & 42.66 \\
\hline UTD & 408.4 & 464.1 & 524.9 & 603.8 & 659.4 & 776.14 & 903.1 & 1089 & 1261 & 1405 & 809.5 & 345.3 & 42.65 \\
\hline VIJ & 378.8 & 445.7 & 552.8 & 724.7 & 717.2 & 817.6 & 984 & 1166 & 1385 & 1500 & 867.2 & 384.7 & 44.37 \\
\hline SBI & 697.7 & 759.1 & 867.1 & 963 & 1085 & 1245.5 & 1469 & 1882 & 2177 & 2675 & 1382 & 663.7 & 48.02 \\
\hline SBBJ & 360.9 & 412.7 & 467.9 & 547.1 & 624.7 & 706.54 & 850.7 & 992.4 & 1153 & 1307 & 742.3 & 324 & 43.64 \\
\hline SBH & 446.5 & 529.9 & 642.4 & 724.5 & 782 & 904.75 & 1040 & 1242 & 1433 & 1690 & 943.5 & 405.5 & 42.98 \\
\hline INDO & 377.9 & 440.5 & 498.3 & 584.1 & 684.7 & 751.88 & 888.9 & 1041 & 1274 & 1638 & 817.9 & 401.4 & 49.07 \\
\hline SBM & 353 & 436.2 & 508.8 & 620.2 & 698.2 & 783.84 & 848.6 & 962.8 & 1118 & 1262 & 759.2 & 295.7 & 38.95 \\
\hline SBP & 483 & 571.1 & 718.7 & 827.7 & 948.2 & 1034.2 & 1094 & 1243 & 1426 & 1601 & 994.7 & 361.2 & 36.31 \\
\hline SBS & 424.1 & 496.8 & 590.8 & 709.8 & 833.7 & 973.68 & 1040 & 1195 & 1429 & 1638 & 933.1 & 401.6 & 43.03 \\
\hline SBT & 445.6 & 521.6 & 634.7 & 741.5 & 833.2 & 988.38 & 1132 & 1304 & 1527 & 1725 & 985.2 & 431.3 & 43.78 \\
\hline
\end{tabular}




\begin{tabular}{|c|c|c|c|c|c|c|c|c|c|c|c|c|c|}
\hline Name of banks & 2001-02 & $2002-03$ & 2003-04 & 2004-05 & 2005-06 & 2006-07 & 2007-08 & 2008-09 & 2009-10 & 2010-11 & Mean & SD & $\mathrm{CV}$ \\
\hline ALLA & 1184 & 1328 & 1627 & 2089 & 2426 & 2889 & 3323 & 3760 & 4637 & 5459 & 2872 & 1430 & 49.78 \\
\hline AND & 1730 & 1915 & 2034 & 2359 & 2797 & 3216 & 3619 & 4147 & 4990 & 5647 & 3245 & 1345 & 41.45 \\
\hline BOB & 2307 & 2413 & 2711 & 2971 & 3415 & 4506 & 5244 & 6474 & 7657 & 8936 & 4663 & 2350 & 50.4 \\
\hline BOI & 2343 & 2505 & 2771 & 3012 & 3551 & 4359 & 5164 & 6224 & 7100 & 8493 & 4552 & 2130 & 46.8 \\
\hline $\mathrm{BOM}$ & 1566 & 1800 & 2073 & 2234 & 2070 & 2522 & 3037 & 3677 & 4357 & 4352 & 2769 & 1035 & 37.36 \\
\hline CAN & 2658 & 2974 & 3497 & 3858 & 4613 & 5523 & 5753 & 6838 & 7704 & 9026 & 5244 & 2120 & 40.42 \\
\hline CBI & 1513 & 1641 & 1786 & 1931 & 2125 & 2592 & 3335 & 3731 & 4532 & 4811 & 2800 & 1224 & 43.71 \\
\hline CORP & 2872 & 3176 & 3212 & 3505 & 3937 & 4701 & 5650 & 7013 & 8029 & 8565 & 5066 & 2130 & 42.04 \\
\hline DENA & 1353 & 1453 & 1617 & 1790 & 2105 & 2440 & 3196 & 3636 & 4198 & 4974 & 2676 & 1262 & 47.14 \\
\hline IND & 1721 & 1963 & 2213 & 2519 & 2911 & 3263 & 3956 & 4415 & 5019 & 5679 & 3366 & 1353 & 40.19 \\
\hline IOB & 2198 & 2561 & 2837 & 2947 & 3329 & 3847 & 4551 & 5217 & 5518 & 6631 & 3964 & 1462 & 36.9 \\
\hline $\mathrm{OBC}$ & 2946 & 3014 & 3522 & 4235 & 4373 & 5027 & 5885 & 7021 & 7975 & 8584 & 5258 & 2034 & 38.68 \\
\hline PSB & 1660 & 1754 & 1795 & 1862 & 2082 & 2289 & 2861 & 3802 & 5355 & 6189 & 2965 & 1626 & 54.86 \\
\hline PNB & 1663 & 1878 & 2186 & 2552 & 2944 & 1909 & 3901 & 4494 & 4985 & 5766 & 3228 & 1461 & 45.27 \\
\hline SYND & 1631 & 1761 & 2410 & 2573 & 2673 & 3700 & 4388 & 5201 & 5070 & 5437 & 3485 & 1460 & 41.9 \\
\hline UCO Bank & 1565 & 1834 & 2286 & 2865 & 3128 & 3500 & 4075 & 4844 & 5688 & 6586 & 3637 & 1660 & 45.64 \\
\hline UNI & 1967 & 2215 & 2503 & 3015 & 3559 & 3861 & 4399 & 5422 & 6062 & 6713 & 3972 & 1651 & 41.57 \\
\hline UTD & 1504 & 1619 & 1748 & 1935 & 2219 & 2805 & 3353 & 3759 & 4445 & 4874 & 2826 & 1224 & 43.33 \\
\hline VIJ & 1773 & 2027 & 2427 & 2815 & 3002 & 3845 & 4563 & 4953 & 5348 & 6104 & 3686 & 1499 & 40.68 \\
\hline SBI & 2978 & 3276 & 3525 & 4009 & 4124 & 4677 & 5233 & 6431 & 6363 & 6818 & 4743 & 1402 & 29.56 \\
\hline SBBJ & 1472 & 1675 & 1946 & 2310 & 2607 & 3374 & 4013 & 4561 & 5349 & 5970 & 3328 & 1588 & 47.73 \\
\hline SBH & 1966 & 2304 & 2689 & 3114 & 3600 & 4301 & 5006 & 6057 & 6158 & 6740 & 4194 & 1725 & 41.15 \\
\hline INDO & 1890 & 2160 & 2395 & 3138 & 3787 & 4489 & 5381 & 6028 & 6488 & & 3973 & 1719 & 43.28 \\
\hline SBM & 1411 & 1480 & 1785 & 2146 & 2554 & 3420 & 4199 & 4884 & 5643 & 6114 & 3364 & 1756 & 52.22 \\
\hline SBP & 1929 & 2421 & 3025 & 3533 & 4456 & 5115 & 6087 & 7093 & 7286 & 6753 & 4770 & 1994 & 41.8 \\
\hline SBS & 1850 & 2187 & 2554 & 3010 & 3249 & 3497 & 3515 & & & & 2837 & 655 & 23.07 \\
\hline SBT & 1997 & 2374 & 2952 & 3602 & 3768 & 4401 & 4979 & 5791 & 6803 & 7343 & 4401 & 1817 & 41.29 \\
\hline
\end{tabular}


Advances per branch

(Rs.in lakh)

\begin{tabular}{|c|c|c|c|c|c|c|c|c|c|c|c|c|c|}
\hline Name of banks & $1991-92$ & $1992-93$ & 1993-94 & $1994-95$ & $1995-96$ & $1996-97$ & $1997-98$ & 1998-99 & 1999-00 & $2000-01$ & Mean & SD & $\mathrm{CV}$ \\
\hline ALLA & 190 & 219 & 202 & 228 & 259 & 265 & 305 & 371 & 435 & 504 & 297.9 & 106 & 35.6 \\
\hline AND & 200 & 199 & 199 & 248 & 264 & 298 & 336 & 450 & 548 & 728 & 347 & 176 & 50.8 \\
\hline BOB & 443 & 489 & 520 & 605 & 647 & 663 & 785 & 820 & 920 & 1402 & 729.4 & 281 & 38.5 \\
\hline BOI & 535 & 485 & 457 & 504 & 636 & 741 & 883 & 934 & 996 & 1270 & 744 & 270 & 36.3 \\
\hline BOM & 162 & 164 & 160 & 206 & 237 & 271 & 312 & 346 & 440 & 547 & 284.4 & 130 & 45.6 \\
\hline CAN & 395 & 393 & 397 & 509 & 597 & 637 & 728 & 821 & 982 & 1157 & 661.6 & 262 & 39.7 \\
\hline CBI & 223 & 124 & 196 & 254 & 289 & 285 & 346 & 414 & 510 & 606 & 324.5 & 148 & 45.6 \\
\hline CORP & 229 & 275 & 302 & 420 & 487 & 595 & 741 & 1019 & 1200 & 1329 & 659.8 & 398 & 60.4 \\
\hline DENA & 152 & 178 & 193 & 254 & 300 & 354 & 445 & 549 & 608 & 596 & 362.8 & 176 & 48.6 \\
\hline IND & 474 & 563 & 481 & 547 & 534 & 462 & 486 & 501 & 548 & 663 & 526 & 60 & 11.4 \\
\hline IOB & 398 & 411 & 402 & 493 & 554 & 529 & 628 & 722 & 812 & 913 & 586.2 & 180 & 30.7 \\
\hline $\mathrm{OBC}$ & 331 & 401 & 440 & 571 & 666 & 647 & 751 & 857 & 1019 & 1188 & 687.3 & 275 & 40 \\
\hline PSB & 199 & 203 & 248 & 353 & 399 & 396 & 448 & 565 & 647 & 694 & 415.3 & 176 & 42.4 \\
\hline PNB & 273 & 321 & 268 & 314 & 340 & 374 & 423 & 498 & 586 & 723 & 412 & 149 & 36.1 \\
\hline SYND & 249 & 271 & 256 & 282 & 340 & 362 & 456 & 558 & 717 & 757 & 424.7 & 191 & 45 \\
\hline UCO Bank & 362 & 325 & 255 & 272 & 276 & 272 & 311 & 347 & 432 & 586 & 343.8 & 101 & 29.3 \\
\hline UNI & 199 & 240 & 272 & 373 & 433 & 452 & 492 & 515 & 684 & 853 & 451.4 & 202 & 44.8 \\
\hline UTD & 214 & 221 & 190 & 211 & 214 & 227 & 253 & 288 & 343 & 436 & 259.7 & 77 & 29.6 \\
\hline VIJ & 213 & 216 & 235 & 291 & 293 & 296 & 386 & 453 & 560 & 679 & 362.4 & 157 & 43.4 \\
\hline SBI & 514 & 546 & 471 & 549 & 673 & 700 & 832 & 917 & 1085 & 1251 & 753.8 & 263 & 34.9 \\
\hline SBBJ & 215 & 241 & 242 & 286 & 328 & 394 & 477 & 492 & 559 & 654 & 389.1 & 151 & 38.8 \\
\hline SBH & 275 & 307 & 317 & 430 & 498 & 505 & 556 & 624 & 697 & 808 & 501.6 & 176 & 35 \\
\hline INDO & 247 & 272 & 289 & 355 & 408 & 432 & 502 & 548 & 710 & 838 & 460.2 & 194 & 42.2 \\
\hline SBM & 214 & 257 & 261 & 339 & 375 & 432 & 468 & 516 & 589 & 711 & 416.3 & 159 & 38.2 \\
\hline SBP & 306 & 348 & 353 & 433 & 505 & 542 & 581 & 676 & 809 & 945 & 549.7 & 210 & 38.1 \\
\hline SBS & 285 & 311 & 316 & 415 & 481 & 567 & 625 & 680 & 792 & 883 & 535.5 & 209 & 39.1 \\
\hline SBT & 244 & 308 & 357 & 484 & 514 & 559 & 606 & 640 & 769 & 953 & 543.6 & 215 & 39.6 \\
\hline
\end{tabular}




\begin{tabular}{|c|c|c|c|c|c|c|c|c|c|c|c|c|c|}
\hline Name of banks & 2001-02 & $2002-03$ & 2003-04 & 2004-05 & 2005-06 & 2006-07 & 2007-08 & 2008-09 & $2009-10$ & 2010-11 & Mean & SD & $\mathrm{CV}$ \\
\hline ALLA & 574 & 654 & 793 & 1084 & 1458 & 2003 & 2307 & 2602 & 3131 & 3875 & 1848 & 1128 & 61.04 \\
\hline AND & 905 & 1047 & 1142 & 1500 & 1822 & 2164 & 2506 & 3082 & 3604 & 4377 & 2215 & 1171 & 52.87 \\
\hline BOB & 1257 & 1284 & 1322 & 1585 & 2184 & 3017 & 3681 & 4820 & 5560 & 6690 & 3140 & 1978 & 63 \\
\hline BOI & 1504 & 1666 & 1790 & 2140 & 2464 & 3089 & 3906 & 4689 & 5207 & 6056 & 3251 & 1625 & 49.99 \\
\hline BOM & 676 & 772 & 919 & 1012 & 1267 & 1704 & 2130 & 2413 & 2775 & 3052 & 1672 & 872 & 52.18 \\
\hline CAN & 1375 & 1670 & 1929 & 2405 & 3137 & 3821 & 4004 & 5057 & 5559 & 65.2 & 2902 & 1729 & 59.58 \\
\hline CBI & 683 & 72.2 & 729 & 867 & 1198 & 1622 & 2207 & 2430 & 2946 & 34.8 & 1279 & 996 & 77.85 \\
\hline CORP & 1667 & 1759 & 1924 & 2387 & 2870 & 3324 & 3994 & 4598 & 5472 & 6372 & 3437 & 1636 & 47.61 \\
\hline DENA & 663 & 743 & 829 & 1007 & 1268 & 1613 & 2168 & 2439 & 2900 & 3472 & 1710 & 987 & 57.71 \\
\hline IND & 781 & 892 & 1027 & 1330 & 1604 & 2014 & 2582 & 3126 & 3535 & 4039 & 2093 & 1169 & 55.86 \\
\hline IOB & 1048 & 1218 & 1388 & 1679 & 2290 & 2633 & 3261 & 3902 & 3934 & 5107 & 2646 & 1371 & 51.8 \\
\hline $\mathrm{OBC}$ & 1464 & 1585 & 1943 & 2239 & 2925 & 3467 & 4124 & 4889 & 5536 & 5920 & 3409 & 1647 & 48.3 \\
\hline PSB & 742 & 781 & 793 & 831 & 1120 & 1391 & 2113 & 2699 & 3555 & 4418 & 1844 & 1315 & 71.3 \\
\hline PNB & 891 & 996 & 1174 & 1494 & 1835 & 2345 & 2801 & 3314 & 3731 & 4461 & 2304 & 1239 & 53.77 \\
\hline SYND & 851 & 937 & 1168 & 1486 & 1818 & 2432 & 2953 & 3659 & 3917 & 4282 & 2350 & 1289 & 54.83 \\
\hline UCO Bank & 746 & 932 & 1201 & 1601 & 2143 & 2536 & 2809 & 3325 & 3834 & 4491 & 2362 & 1269 & 53.72 \\
\hline UNI & 1057 & 1263 & 1457 & 1955 & 2564 & 2828 & 3149 & 3774 & 4254 & 5006 & 2731 & 1332 & 48.79 \\
\hline UTD & 523 & 566 & 612 & 869 & 1178 & 1672 & 1988 & 2439 & 2759 & 3350 & 1596 & 1010 & 63.31 \\
\hline VIJ & 748 & 935 & 1275 & 1575 & 1805 & 2477 & 3015 & 3221 & 3586 & 4060 & 2270 & 1165 & 51.34 \\
\hline SBI & 1330 & 1524 & 1747 & 2210 & 2841 & 3623 & 4059 & 4701 & 5000 & 5524 & 3256 & 1539 & 47.26 \\
\hline SBBJ & 749 & 854 & 1069 & 1457 & 1911 & 2432 & 2950 & 3471 & 4091 & 4568 & 2355 & 1375 & 58.36 \\
\hline SBH & 952 & 1081 & 1310 & 1679 & 2208 & 2913 & 3581 & 4237 & 4476 & 4922 & 2736 & 1497 & 54.7 \\
\hline INDO & 1024 & 1214 & 1473 & 2055 & 2699 & 3450 & 3970 & 4598 & 5016 & & 2833 & 1497 & 52.84 \\
\hline SBM & 814 & 864 & 1016 & 1387 & 1834 & 2557 & 3215 & 3801 & 4287 & 4813 & 2459 & 1496 & 60.84 \\
\hline SBP & 1200 & 1456 & 1761 & 2048 & 2926 & 3756 & 4561 & 5152 & 5231 & 5103 & 3319 & 1636 & 49.28 \\
\hline SBS & 1000 & 1123 & 1254 & 1602 & 1982 & 2452 & 2661 & & & & 1725 & 658 & 38.13 \\
\hline SBT & 1103 & 1367 & 1667 & 2216 & 2734 & 3521 & 3963 & 4491 & 5142 & 5814 & 3202 & 1641 & 51.27 \\
\hline
\end{tabular}


Net Profit per branch

(Rs.in lakh)

\begin{tabular}{|c|c|c|c|c|c|c|c|c|c|c|c|c|c|}
\hline Name of banks & 1991-92 & $1992-93$ & 1993-94 & $1994-95$ & $1995-96$ & 1996-97 & 1997-98 & $1998-99$ & 1999-00 & $2000-01$ & Mean & SD & $\mathrm{CV}$ \\
\hline ALLA & 1.55 & -5.88 & -19.9 & -4.1 & 0.32 & 3.44 & 6.88 & 7.17 & 3.66 & 2.1 & -0.48 & 8.01 & -1667 \\
\hline AND & 0.84 & -14.7 & -16.7 & -4.54 & 1.12 & 3.7 & 7.64 & 8.95 & 11.8 & 11.9 & 1.00 & 10.2 & 1021 \\
\hline BOB & 4.02 & 0.33 & 2.71 & 7.16 & 8.4 & 11.1 & 18.2 & 16.4 & 19 & 10.3 & 9.75 & 6.51 & 66.8 \\
\hline BOI & 2.43 & -13.9 & -45.6 & 2.06 & 11.2 & 14.5 & 14.6 & 8 & 6.83 & 9.94 & 1.02 & 18.4 & 1798 \\
\hline BOM & 0.36 & -17.4 & -26.3 & -3.61 & 1.14 & 4.1 & 4.82 & 4.42 & 7.54 & 3.71 & -2.12 & 11 & -520 \\
\hline CAN & 7.75 & 1.27 & 5.74 & 9.55 & 11.5 & 6.5 & 8.78 & 9.46 & 9.85 & 11.9 & 8.23 & 3.13 & 38 \\
\hline CBI & 1 & -12.6 & -23.3 & -2.73 & -2.4 & 4.89 & 5.67 & 4.73 & 4.86 & 1.49 & -1.83 & 9.33 & -509 \\
\hline CORP & 1.13 & 0.89 & 5.73 & 14.8 & 21 & 24.7 & 28.7 & 31.1 & 35.9 & 40.2 & 20.41 & 14.3 & 69.9 \\
\hline DENA & 0.82 & -8.06 & -6.24 & 2.65 & 4.59 & 6.39 & 9.08 & 9.44 & 5.37 & -23 & 0.13 & 9.93 & 7136 \\
\hline IND & 2.71 & 0.51 & -27.8 & 0.97 & -91 & -26 & -20 & -52 & 28.5 & -19 & -20.3 & 33.1 & -163 \\
\hline IOB & 0.69 & -57.6 & -26.4 & 0.74 & 0.22 & 7.66 & 8.19 & 3.95 & 2.83 & 8.08 & -5.16 & 21 & -406 \\
\hline $\mathrm{OBC}$ & 5.07 & 3.8 & 5.49 & 18.4 & 24.7 & 23.8 & 25 & 25.6 & 30.5 & 21.8 & 18.41 & 9.88 & 53.7 \\
\hline PSB & 0.14 & -25.5 & -25.7 & -1.01 & -19 & 2.84 & 9.14 & 7.74 & 8.35 & 1.78 & -4.10 & 13.8 & -337 \\
\hline PNB & 3.67 & 1.23 & 2.04 & 2.32 & -2.6 & 6.32 & 12.6 & 9.74 & 10.6 & 12 & 5.78 & 5.21 & 90 \\
\hline SYND & 0.26 & -43 & -19.2 & -5.87 & 1.26 & 4.16 & 5.44 & 8.54 & 12.7 & 13.6 & -2.21 & 17.2 & -776 \\
\hline UCO Bank & -1.17 & -24.8 & -30.4 & -4.68 & -13 & -9.8 & -5.3 & -3.8 & 2.08 & 1.92 & -8.90 & 11 & -123 \\
\hline UNI & 1.57 & 0.59 & 2.64 & 5.55 & 3.99 & 10.6 & 12 & 7.56 & 4.74 & 7.57 & 5.68 & 3.76 & 66.1 \\
\hline UTD & 0.53 & -21 & 46.4 & 14.8 & 17.6 & -8.6 & 0.75 & 1.1 & 2.36 & 1.46 & 5.53 & 17.9 & 324 \\
\hline VIJ & 0.28 & -13.2 & 0.51 & 3.95 & -30 & 2.28 & 2.75 & 3.64 & 6.31 & 8.4 & -1.51 & 11.6 & -765 \\
\hline SBI & 2.03 & 2.43 & 3.12 & 8.09 & 9.36 & 15.2 & 20.9 & 11.4 & 22.7 & 17.7 & 11.29 & 7.62 & 67.5 \\
\hline SBBJ & 1.43 & 1.55 & 0.95 & 1.09 & 3.49 & 5.24 & 11.7 & 11.8 & 15.3 & 13.3 & 6.59 & 5.78 & 87.7 \\
\hline SBH & 1.83 & 2.33 & 3.2 & 5.73 & 6.42 & 6.5 & 11.7 & 13 & 14.6 & 17.1 & 8.24 & 5.46 & 66.2 \\
\hline INDO & 0.9 & 0.89 & 1.13 & 2.56 & 3.33 & 4.57 & 7.39 & 8.02 & 11.3 & 15.6 & 5.57 & 4.97 & 89.2 \\
\hline SBM & 0.79 & 0.78 & 0.38 & 0.57 & 4.78 & 7.18 & 9.07 & 5.8 & 8.13 & 4.27 & 4.17 & 3.37 & 80.7 \\
\hline SBP & 5.22 & 3.34 & 4.64 & 5.16 & 7.94 & 8.44 & 20.2 & 14.2 & 18.3 & 22.3 & 10.98 & 7.14 & 65 \\
\hline SBS & 1.83 & 1.45 & 1.67 & 3.79 & -61 & 18.7 & 20.3 & 6.34 & 21.4 & 3.37 & 1.78 & 23.5 & 1319 \\
\hline SBT & 0.81 & 1.3 & 1.43 & 3.25 & 3.99 & 6.12 & 9.55 & 6.52 & 9.96 & 14.5 & 5.74 & 4.5 & 78.3 \\
\hline
\end{tabular}




\begin{tabular}{|c|c|c|c|c|c|c|c|c|c|c|c|c|c|}
\hline Name of banks & 2001-02 & $2002-03$ & 2003-04 & 2004-05 & 2005-06 & 2006-07 & 2007-08 & 2008-09 & $2009-10$ & 2010-11 & Mean & $\mathrm{SD}$ & $\mathrm{CV}$ \\
\hline ALLA & 4.19 & 8.65 & 23.9 & 27.8 & 35.3 & 36.4 & 45.2 & 34 & 52.7 & 58.9 & 32.7 & 17.5 & 53.52 \\
\hline AND & 18.9 & 36.6 & 41.1 & 44.5 & 40 & 41.7 & 42.1 & 45.6 & 67.2 & 77.6 & 45.5 & 16.2 & 35.58 \\
\hline BOB & 20.4 & 28.1 & 35.9 & 24.7 & 30.1 & 37 & 49.5 & 74.9 & 97.2 & 124 & 52.2 & 35 & 67.12 \\
\hline BOI & 19.8 & 33.3 & 39.4 & 13 & 26.5 & 40.8 & 69.2 & 98.7 & 53.8 & 70.7 & 46.5 & 26.6 & 57.18 \\
\hline BOM & 11.9 & 18 & 23.9 & 13.7 & 3.91 & 20.2 & 23.9 & 26.4 & 30.3 & 21.5 & 19.4 & 7.76 & 40.09 \\
\hline CAN & 30.8 & 42 & 54.2 & 44.2 & 53 & 55.1 & 58.4 & 75.8 & 99.2 & 124 & 63.6 & 28.4 & 44.55 \\
\hline CBI & 5.24 & 9.8 & 19.7 & 11.4 & 8.23 & 15.6 & 16.6 & 16.2 & 29.6 & 33.6 & 16.6 & 9.08 & 54.66 \\
\hline CORP & 46.8 & 60.8 & 69.8 & 51.8 & 53.2 & 59.5 & 74.9 & 84.6 & 101 & 104 & 70.6 & 20.3 & 28.7 \\
\hline DENA & 1 & 10.1 & 20.3 & 5.43 & 6.51 & 17.8 & 33.9 & 35.7 & 41.8 & 47.4 & 22 & 16.6 & 75.57 \\
\hline IND & 2.38 & 13.7 & 29.5 & 29.6 & 36 & 52.7 & 65.4 & 75.7 & 88.5 & 92 & 48.5 & 31.2 & 64.23 \\
\hline IOB & 15.9 & 29 & 35.1 & 43.4 & 51.6 & 56.4 & 64.9 & 69.1 & 35.2 & 49 & 45 & 16.5 & 36.8 \\
\hline $\mathrm{OBC}$ & 33.1 & 46.2 & 67.7 & 67.3 & 48.5 & 45.6 & 63.6 & 63.6 & 75.2 & 92.8 & 60.4 & 17.3 & 28.65 \\
\hline PSB & 3.06 & 0.59 & 1.17 & -9.34 & 13.3 & 25.9 & 44.1 & 47.3 & 55.4 & 54.5 & 23.6 & 24.9 & 105.7 \\
\hline PNB & 14.6 & 20.9 & 27.6 & 34.9 & 35.4 & 37.4 & 48 & 66.2 & 78.1 & 81.7 & 44.5 & 23.5 & 52.8 \\
\hline SYND & 14.3 & 19.8 & 24.6 & 22.4 & 26.7 & 33.7 & 39.1 & 41 & 35.2 & 42 & 29.9 & 9.64 & 32.28 \\
\hline UCO Bank & 9.59 & 12.1 & 25.4 & 20 & 11.3 & 17.1 & 21 & 27 & 47 & 41.1 & 23.2 & 12.5 & 54.05 \\
\hline UNI & 15.5 & 27.4 & 35.3 & 35.1 & 32.4 & 38.3 & 58.7 & 67.5 & 74 & 69 & 45.3 & 20.2 & 44.66 \\
\hline UTD & 9.13 & 23.5 & 24.2 & 22.9 & 15.5 & 20.2 & 22.8 & 12.7 & 21 & 32.8 & 20.5 & 6.67 & 32.56 \\
\hline VIJ & 15.8 & 23.3 & 47.5 & 41.8 & 13.7 & 33.9 & 34.4 & 23.8 & 43.8 & 43.7 & 32.2 & 12.3 & 38.18 \\
\hline SBI & 26.8 & 34.4 & 40.7 & 47 & 47.8 & 48.8 & 65.5 & 79 & 72.5 & 60.3 & 52.3 & 16.8 & 32.08 \\
\hline SBBJ & 20.8 & 25.6 & 37.5 & 25 & 17.4 & 36.2 & 37.1 & 46.9 & 52.9 & 61.1 & 36 & 14.3 & 39.69 \\
\hline SBH & 25.6 & 33.7 & 42.3 & 27 & 45.2 & 52.4 & 55.6 & 59.7 & 69.4 & 88.7 & 50 & 19.7 & 39.35 \\
\hline INDO & 29.9 & 46.9 & 52 & 30.3 & 31.6 & 42.7 & 51 & 59.3 & 65.2 & & 45.4 & 12.9 & 28.4 \\
\hline SBM & 10.9 & 19 & 28.4 & 32.6 & 33.8 & 38.7 & 48.8 & 50 & 64.7 & 70.8 & 39.8 & 19 & 47.79 \\
\hline SBP & 32.2 & 43.6 & 57.9 & 38.3 & 40 & 47.8 & 51.8 & 62.8 & 62.2 & 64.8 & 50.2 & 11.5 & 22.99 \\
\hline SBS & 20 & 22.4 & 42.4 & 9.82 & 14.1 & 19.3 & 11.3 & & & & 19.9 & 11 & 55.16 \\
\hline SBT & 17.9 & 25.5 & 36.6 & 36.9 & 37.5 & 46.3 & 54.4 & 83.7 & 91.5 & 91.9 & 52.2 & 27.3 & 52.37 \\
\hline
\end{tabular}


Employee Productivity Performance for the period 1991- 2000

\begin{tabular}{|c|c|c|c|}
\hline $\begin{array}{c}\text { Index } \rightarrow \\
\text { Performance } \\
\downarrow\end{array}$ & Deposit per Employee & Advances per & Net Profit per Employee \\
\hline Excellent & $\begin{array}{c}\text { Bank of Baroda, Bank of India, Corporation bank, Oriental bank of } \\
\text { Commerce, Union bank of India }\end{array}$ & $\begin{array}{l}\text { Bank of Baroda, Bank of India, Corporation bank, } \\
\text { Oriental bank of Commerce }\end{array}$ & $\begin{array}{l}\text { Bank of Baroda, Corporation bank, Oriental } \\
\text { bank of Commerce, State bank of Hyderabad, } \\
\text { SBOP }\end{array}$ \\
\hline Good & $\begin{array}{l}\text { Andhra bank, Canara bank, Dena bank, Indian bank, Indian } \\
\text { Oversea bank, Punjab and Sind bank, State bank of Hyderabad. }\end{array}$ & $\begin{array}{c}\text { Canara bank, Dena bank, Indian bank, Indian Oversea } \\
\text { bank, Union bank of India, SBI, State bank of } \\
\text { Hyderabad, SBOP, State bank of Tranvancore }\end{array}$ & $\begin{array}{c}\text { Canara bank, PNB, Union bank of India, United } \\
\text { bank of India, SBI, State bank of Bikaner and } \\
\text { Jaipur, State bank of Indore, state bank of } \\
\text { Mysore, State bank of Tranvancore }\end{array}$ \\
\hline Fair & $\begin{array}{c}\text { Allahabad bank, bank of Maharashtra, Central bank of India, PNB, } \\
\text { United bank of India, Vijaya bank, } \\
\text { SBI, SBOP, State bank of Tranvancore }\end{array}$ & $\begin{array}{l}\text { Allahabad bank, Andhra bank, Punjab and Sind bank, } \\
\text { PNB, State bank of Indore, state bank of Saurashtra }\end{array}$ & $\begin{array}{c}\text { Andhra bank, Bank of India, state bank of } \\
\text { Saurashtra }\end{array}$ \\
\hline Poor & $\begin{array}{c}\text { Syndicate bank, UCO bank, State bank of Bikaner and Jaipur, State } \\
\text { bank of Indore, Mysore and Saurashtra }\end{array}$ & $\begin{array}{c}\text { Bank of Maharashtra, Central bank of India, Syndicate } \\
\text { bank, UCO bank, United bank of India, Vijaya bank, } \\
\text { State bank of Bikaner and Jaipur, state bank of } \\
\text { Mysore }\end{array}$ & $\begin{array}{c}\text { Allahabad bank, Bank of Maharashtra, Central } \\
\text { bank of India, Dena bank, Indian bank, Indian } \\
\text { Oversea bank, Punjab and Sind bank, Syndicate } \\
\text { bank, UCO bank, Vijaya bank }\end{array}$ \\
\hline
\end{tabular}

\section{Employee Productivity Performance for the period 2001-2010}

\begin{tabular}{|c|c|c|c|}
\hline $\begin{array}{l}\text { Index } \rightarrow \\
\text { Performance }\end{array}$ & Deposit per Employee & Advances per & Net Profit per Employee \\
\hline Excellent & $\begin{array}{c}\text { Bank of Baroda, Corporation bank, Oriental bank of Commerce, } \\
\text { Union bank of India }\end{array}$ & $\begin{array}{c}\text { Bank of Baroda, Bank of India, Corporation bank, } \\
\text { Oriental bank of Commerce, Union bank of India, } \\
\text { SBOP }\end{array}$ & $\begin{array}{l}\text { Andhra bank, Bank of Baroda, Canara bank, } \\
\text { Corporation bank, Oriental bank of Commerce, } \\
\text { Union bank of India, State bank of Hyderabad }\end{array}$ \\
\hline Good & $\begin{array}{c}\text { Allahabad bank, Andhra bank, Bank of India, Canara bank, Dena } \\
\text { bank, Vijaya bank, State bank of Hyderabad, SBOP }\end{array}$ & $\begin{array}{l}\text { Andhra bank, Canara bank, Syndicate bank, Vijaya } \\
\text { bank, State bank of Hyderabad, State bank of Indore }\end{array}$ & $\begin{array}{l}\text { Allahabad bank, Bank of India, Indian bank, } \\
\text { Indian Oversea bank, PNB, State bank of } \\
\text { Indore, SBOP, State bank of Tranvancore }\end{array}$ \\
\hline Fair & $\begin{array}{c}\text { Bank of Maharashtra, Central bank of India, Indian Oversea bank, } \\
\text { Punjab and Sind bank, Syndicate bank, UCO bank, State bank of } \\
\text { Indore, State bank of Tranvancore }\end{array}$ & $\begin{array}{c}\text { Allahabad bank, Dena bank, Indian Oversea bank, } \\
\text { Punjab and Sind bank, PNB, UCO bank, SBI, State } \\
\text { bank of Tranvancore }\end{array}$ & $\begin{array}{c}\text { Dena bank, Vijaya bank, SBI, State bank of } \\
\text { Bikaner and Jaipur, Mysore }\end{array}$ \\
\hline Poor & $\begin{array}{l}\text { Indian bank, PNB, United bank of India, SBI, State bank of Bikaner } \\
\text { and Jaipur, state bank of Mysore, state bank of Saurashtra }\end{array}$ & $\begin{array}{l}\text { Bank of Maharashtra, Central bank of India, Indian } \\
\text { bank, United bank of India, State bank of Bikaner and } \\
\text { Jaipur, state bank of Mysore, state bank of Saurashtra }\end{array}$ & $\begin{array}{c}\text { Bank of Maharashtra, Central bank of India, } \\
\text { Punjab and Sind bank, Syndicate bank, UCO } \\
\text { bank, United bank of India, state bank of } \\
\text { Saurashtra }\end{array}$ \\
\hline
\end{tabular}

\section{Branch Productivity Performance for the period 1991-2000}




\begin{tabular}{|c|c|c|c|}
\hline $\begin{array}{c}\text { Index } \rightarrow \\
\text { Performance } \\
\downarrow\end{array}$ & Deposit per Branch & Advances per Branch \\
\hline Excellent & $\begin{array}{c}\text { Bank of Baroda, Bank of India, Canara bank, Corporation bank, } \\
\text { Indian Oversea bank, Oriental bank of Commerce, SBI }\end{array}$ & $\begin{array}{c}\text { Net Profit per Branch } \\
\text { Corporation bank, Indian Oversea, bank, Oriental bank } \\
\text { of Commerce, SBI }\end{array}$ & $\begin{array}{c}\text { Bank of Baroda, Canara bank, Corporation bank, } \\
\text { India, United bank of India, SBI, State bank of } \\
\text { Bikaner and Jaipur, State bank of Hyderabad, State } \\
\text { bank of Indore, SBOP, State bank of Tranvancore }\end{array}$ \\
\hline Good & $\begin{array}{c}\text { Indian bank, Union bank of India, SBOP, State bank of } \\
\text { Tranvancore }\end{array}$ & $\begin{array}{c}\text { Indian bank, State bank of Hyderabad, SBOP, } \\
\text { Saurashtra, State bank of Tranvancore }\end{array}$ \\
\hline Fair & $\begin{array}{c}\text { Andhra bank, Punjab and Sind bank, PNB, Syndicate bank, } \\
\text { Vijaya bank, State bank of Hyderabad, state bank of } \\
\text { Saurashtra }\end{array}$ & $\begin{array}{c}\text { Punjab and Sind bank, PNB, Syndicate bank, Union } \\
\text { bank of India, State bank of Bikaner and Jaipur, State } \\
\text { bank of Indore, state bank of Mysore }\end{array}$ & $\begin{array}{c}\text { Andhra bank, Bank of India, Dena bank, state bank } \\
\text { of Saurashtra }\end{array}$ \\
\hline Poor & $\begin{array}{c}\text { Allahabad bank, Bank of Maharashtra, Central bank of India, } \\
\text { Dena bank, UCO bank, United bank of India, State bank of } \\
\text { Bikaner and Jaipur, State bank of Indore, state bank of Mysore }\end{array}$ & $\begin{array}{c}\text { Allahabad bank Andhra bank, bank of Maharashtra, } \\
\text { Central bank of India, Dena bank, UCO bank, United } \\
\text { bank of India, Vijaya bank }\end{array}$ & $\begin{array}{c}\text { Allahabad bank, bank of Maharashtra, Central bank } \\
\text { of India, Indian bank, Indian Oversea bank, Punjab } \\
\text { and Sind bank, Syndicate bank, UCO bank, Vijaya } \\
\text { bank }\end{array}$ \\
\hline
\end{tabular}

\section{Branch Productivity Performance for the period 2001-2010}

\begin{tabular}{|c|c|c|c|}
\hline $\begin{array}{c}\text { Index } \rightarrow \\
\text { Performance } \\
\downarrow\end{array}$ & Deposit per Branch & Advances per Branch \\
\hline Excellent & $\begin{array}{c}\text { Bank of Baroda, Bank of India, Canara bank, Corporation bank, } \\
\text { Oriental bank of Commerce, SBI, SBOP, State bank of } \\
\text { Tranvancore }\end{array}$ & $\begin{array}{c}\text { Bank of Baroda, Bank of India, Corporation bank, } \\
\text { Oriental bank of Commerce, SBI, SBOP, State bank of } \\
\text { Tranvancore }\end{array}$ & $\begin{array}{c}\text { Bank of Baroda, Canara bank, Corporation bank, } \\
\text { Oriental bank of Commerce, SBI, State bank of } \\
\text { Tranvancore }\end{array}$ \\
\hline Good & Indian Oversea bank, Union bank of India, State bank of \\
Hyderabad, State bank of Indore & $\begin{array}{c}\text { Canara bank, Indian Oversea bank, Union bank of } \\
\text { India, State bank of Hyderabad, State bank of Indore }\end{array}$ & $\begin{array}{c}\text { Andhra bank, Bank of India, Indian bank, Indian } \\
\text { of Hyderabad, State bank of Indore, SBOP }\end{array}$ \\
\hline Fair & $\begin{array}{c}\text { Andhra bank, Indian bank, PNB, Syndicate bank, UCO bank, } \\
\text { Vijaya bank, State bank of Bikaner and Jaipur, state bank of } \\
\text { Mysore }\end{array}$ & $\begin{array}{c}\text { Andhra bank, Indian bank, PNB, Syndicate bank, } \\
\text { UCO bank, Vijaya bank, State bank of Bikaner and } \\
\text { Jaipur, state bank of Mysore }\end{array}$ & $\begin{array}{c}\text { Allahabad bank, Vijaya bank, State bank of Bikaner } \\
\text { and Jaipur, state bank of Mysore }\end{array}$ \\
\hline Poor & $\begin{array}{c}\text { Allahabad bank, Bank of Maharashtra, Central bank of India, } \\
\text { Dena bank, Punjab and Sind bank, United bank of India, state } \\
\text { bank of Saurashtra }\end{array}$ & $\begin{array}{c}\text { Allahabad bank, Bank of Maharashtra, Central bank of } \\
\text { India, Dena bank, Punjab and Sind bank, United bank } \\
\text { of India, state bank of Saurashtra }\end{array}$ & $\begin{array}{c}\text { Bank of Maharashtra, Central bank of India, Dena } \\
\text { bank, Punjab and Sind bank, Syndicate bank, UCO } \\
\text { bank, United bank of India, state bank of Saurashtra }\end{array}$ \\
\hline
\end{tabular}




\begin{tabular}{|c|c|c|}
\hline No of Banks & Legends & Name of the Banks \\
\hline 1 & ALLA & Allahabad bank \\
\hline 2 & AND & Andhra bank \\
\hline 3 & ВОВ & Bank of Baroda \\
\hline 4 & BOI & Bank of India \\
\hline 5 & ВОМ & Bank of Maharashtra \\
\hline 6 & CAN & Canara bank \\
\hline 7 & CBI & Central Bank of India \\
\hline 8 & CORP & Corporation bank \\
\hline 9 & DENA & Dena bank \\
\hline 10 & IND & Indian bank \\
\hline 11 & IOB & Indian Overseas bank \\
\hline 12 & ОВC & Oriental Bank of Commerce \\
\hline 13 & PSB & Punjab \& Sind bank \\
\hline 14 & PNB & Punjab National Bank \\
\hline 15 & SYND & Syndicate bank \\
\hline 16 & UCO Bank & UCO bank \\
\hline 17 & UNI & Union Bank of India \\
\hline 18 & UTD & United Bank of India \\
\hline 19 & VIJ & Vijaya bank \\
\hline 20 & SBI & State Bank of India \\
\hline 21 & SBBJ & Bank of Bikaner \& Jaipur \\
\hline 22 & SBH & State bank of Hyderabad \\
\hline 23 & INDO & State bank of Indore \\
\hline 24 & SBM & State bank of Mysore \\
\hline 25 & SBP & State bank of Patiala \\
\hline 26 & SBS & State bank of Saurashtra \\
\hline 27 & SBT & State bank of Tranvancore \\
\hline
\end{tabular}

\section{REFERENCES}

[1] Narasimhan Committee. (1991), "Report of the Committee on the Financial System," Government of India.

[2] Sabi, M. (1991), "Comparative analysis of Domestic and Foreign bank operations in Hungary", Journal of Comparative Economics, Vol.22, No.2, pp179-188.

[3] D' Souza, Errol.(2002), “ How well have Public Sector Banks Done- A Note," Economic and Political Weekly, Vol.37, pp 867-870.

[4] Bhattacharya, S. and Urjit P. (2003), "Reform Strategies in India," Conference on India's and China's Experience with Reforms and Growth.

[5] Kumbhakar, Subal C \& Sarkar, Subrata, (2003),
"Deregulation, Ownership, and Productivity Growth in the Banking Industry: Evidence from India," Journal of Money, Credit and Banking, Ohio State University Press, Vol. 35(3), pp 403-24.

[6] Shanmugam, K.R. and Das, A.(2004), “Efficiency of Indian Commercial banks during the reform period," Applied Financial Economics, No.14, pp 681-686.

[7] Mohan,R.(2005), "Reforms, Productivity and Efficiency in Banking: The Indian Experience," The Pakistan Development Review, 44:4 (Winter), pp.505-538.

[8] Kumar, S. (2008), “An analysis of efficiency -profitability relationship in Indian Public Sector Banks," Global Business Review 9:1, pp115-129.

[9] Das, S.K. (2010), "Financial Liberalization and Banking Sector Efficiency: The Indian Experience," 12th Money and Finance Conference IGIDR, Mumbai.

[10] Reserve Bank of India (2010), "Report on Trends and Progress of Banking in India," 2009-2010. 